\title{
Angular dimensions of planetary nebulae ${ }^{\star}$
}

\author{
R. Tylenda ${ }^{1}$, N. Siódmiak ${ }^{1}$, S. K. Górny ${ }^{1}$, R. L. M. Corradi ${ }^{2}$, and H. E. Schwarz ${ }^{3}$ \\ 1 N. Copernicus Astronomical Center, Department for Astrophysics, Rabiańska 8, 87-100 Toruń, Poland \\ 2 Isaac Newton Group of Telescopes, Apartado de Correos 321, 38700 Sta. Cruz de La Palma, Spain \\ ${ }^{3}$ CTIO/NOAO, Casilla 603, La Serena, Chile
}

Received 28 May 2002 / Accepted 23 April 2003

\begin{abstract}
We have measured angular dimensions of 312 planetary nebulae from their images obtained in $\mathrm{H} \alpha$ (or $\mathrm{H} \alpha+[\mathrm{NII}]$ ). We have applied three methods of measurements: direct measurements at the $10 \%$ level of the peak surface brightness, Gaussian deconvolution and second-moment deconvolution. The results from the three methods are compared and analysed. We propose a simple deconvolution of the $10 \%$ level measurements which significantly improves the reliability of these measurements for compact and partially resolved nebulae. Gaussian deconvolution gives consistent but somewhat underestimated diameters compared to the $10 \%$ measurements. Second-moment deconvolution gives results in poor agreement with those from the other two methods, especially for poorly resolved nebulae. From the results of measurements and using the conclusions of our analysis we derive the final nebular diameters which should be free from systematic differences between small (partially resolved) and extended (well resolved) objects in our sample.
\end{abstract}

Key words. planetary nebulae: general

\section{Introduction}

The angular dimensions belong to the most fundamental observational data for planetary nebulae $(\mathrm{PNe})$. They are required if one wants to study important PN parameters such as linear dimensions, lifetimes, surface brightnesses. Several methods for determining distances or estimating nebular masses rely on observational measurements of the PN diameters. Also studies of the observed evolution of PNe and their central stars often use the angular PN diameters as one of the crucial observational data.

PN dimensions have been measured since the very begining of research interest in these objects and the list of papers with resultant values is very long. Consequently, for almost all the known PNe we have information about their angular dimensions, see e.g. the ESO-Strasbourg Catalogue (Acker et al. 1992). However the data from many existing catalogues and databases should be used with caution. They have usually been compiled from numerous different sources based on different observational techniques and methods. Therefore the resulting data for different objects are not always comparable. For many $\mathrm{PNe}$ the diameters taken from different observational sources can differ by a factor of a few.

The problem is that different observational techniques and/or different methods for measuring dimensions give

Send offprint requests to: N. Siódmiak,

e-mail: alexan@ncac. torun.pl

$\star$ Table 1 is only available in electronic form at

http://www.edpsciences.org different results for many PNe. In the case of well resolved and fairly symmetric nebulae with a well defined outer rim the problem is simple and different methods give consistent results. Most of the PNe have, however, complex structures, soft outer boundaries, and low surface brightness emission extending to large distances. If one measures the maximum extension of the nebular emission in an image, as it has often been done while determining PN dimensions, than the result depends on how deep the image has been made. A reasonable solution in order to get results comparable for all objects is to measure nebular extension at a given level of surface brightness, e.g. at $10 \%$ of the maximum value.

Another problem arises in the case of small PNe when the nebular diameter is comparable to the angular resolution of the instrument. The observed image is then a convolution of an intrinsic nebular image and the instrumental profile. It has to be deconvolved in order to get a meaningful result. It is usually done using the so-called Gaussian deconvolution. The disadvantage of this method is that one has to adopt a shape of the intrinsic surface brightness distribution and the result depends on this assumption. The problem of determining dimensions of partially resolved nebulae has recently been discussed by van Hoof (2000). He has made a thorough model analysis of different methods for measuring partially resolved nebulae.

The question that arises, and which is important for using the measured dimensions in extensive, systematic studies of $\mathrm{PNe}$, is to what extent the results from different methods are comparable. For instance, do the results from direct measurements at the $10 \%$ contour for extended nebulae mean the 
same as the values from a deconvolution method for compact objects? If the PNe were close to model nebulae studied by van Hoof (2000) the answer would be: yes, they measure more or less the same. However, real nebulae are often much different from the model ones and the answer is not a priori clear. We attempt to answer the above question in this paper.

In recent years, a number of observational surveys for studying PN structures and dimensions have been done. As surveys in radio wavelengths those of Aaquist \& Kwok (1990) and Zijlstra et al. (1989) should be mentioned. Over 400 PNe have been observed in these two surveys, mostly at $5 \mathrm{GHz}$. PN diameters have been determined either from a $10 \%$ contour level (for well resolved nebulae) or from a Gaussian deconvolution (for partially resolved objects). Recent optical surveys can be found in Schwarz et al. (1992), Manchado et al. (1996), and Górny et al. (1999) where about 600 PNe have been observed. However, the PN dimensions given in these three catalogues usually refer to the maximum extension of the recorded emission. As the ratio of the maximum to faintest recorded surface brightness can vary by a large factor from one image to another these diameters do not measure the same for all the objects in the catalogues.

We have measured angular dimensions for $312 \mathrm{PNe}$. One of the goals is to provide a homogeneous set of PN diameters, i.e. values which mean the same, as much as possible, for all the objects in the sample. We have applied direct measurements at $10 \%$ of the peak surface brightness as well as deconvolution methods. The results have been used to investigate reliability of the methods and for comparative studies of the results from different methods. This helped us to obtain a homogeneous set of the PN diameters for the whole sample without important systematic differences between compact and extended nebulae.

\section{Observational material}

The observational material used in this paper is, in principle, the same as in Schwarz et al. (1992) and Górny et al. (1999). These are images of mostly southern PNe taken with the NTT and other telescopes at the ESO. For our measurements we have used only images in $\mathrm{H} \alpha$ (or $\mathrm{H} \alpha+[\mathrm{NII}]$ ). This has been done in order to have a uniform set of data and to avoid possible ionization stratification effects in the results which could have appeared if we had used, for instance, [OIII] images.

\section{Methods of measurements}

We have applied three methods of measuring PN dimensions. First one is a direct measurement of the nebular diameter at a level of $10 \%$ of the peak surface brightness, $\Theta_{10 \%}$, from the PN image. As the nebulae in majority are not spherically symmetric we have measured two diameters along perpendicular axes. One of the axes was directed along the longest extension of the nebular emission.

Second method is the Gaussian deconvolution. The full width at half-maximum (FWHM) values, $\Phi$, have been obtained from a two-dimensional Gaussian fitting to the observed PN image. Before fitting the background has been subtracted from the image. The same applied to field stars allowed us to derive the FWHM of the resolution profile, $\Phi_{\mathrm{b}}$. The deconvolved FWHM diameter, $\Phi_{\mathrm{d}}$, can then be obtained from

$\Phi_{\mathrm{d}}=\sqrt{\Phi^{2}-\Phi_{\mathrm{b}}^{2}}$

In order to get an estimate of the real nebular diameter, $\Phi_{\mathrm{d}}$ has to be multiplied by a conversion factor which is a function of the observational resolution, $\Phi_{\mathrm{d}} / \Phi_{\mathrm{b}}$, and the adopted intrinsic surface brightness distribution of the nebula. Details of calculating the conversion factor for a constant surface brightness disc and constant emissivity shells can be found in van Hoof (2000).

Third method is the so-called second-moment deconvolution. This is an analogous method to the Gaussian deconvolution but the FWHM is determined from the second moment of the surface brightness distribution. The advantage of this method is that, unlike Gaussian deconvolution, the conversion factor is independent of the resolution of the observations. Details of applying second-moment deconvolution can be found in van Hoof (2000).

It is well known that the first method fails for small objects and therefore it is usually applied to well resolved nebulae. The deconvolution methods, on the contrary, are usually applied to small, partially resolved nebulae. In this paper we have, however, attempted to apply all the three methods to all the PNe in our sample. This has been done in order to be able to compare different methods and to study systematic differences between results from them.

\section{Results of measurements}

Table 1 presents the results of direct measurements done on our PN images. Following suggestions of van Hoof (2000) we give raw data from the deconvolution methods, i.e. the deconvolved FWHM, $\Phi_{\mathrm{d}}$, and the image resolution, $\Phi_{\mathrm{b}}$. Our final angular diameters of the PNe are given in Table 2. They are based on the measurements reported in Table 1 as well as on the discussion of the results from different methods presented in Sect. 5.

Column (1) in Table 1 gives the PNG number of the object while its usual name is in Col. (2). Column (3) gives the number of a figure showing the PN image in the original papers: "s" and "g" stand for Schwarz et al. (1992) and Górny et al. (1999), respectively. The deconvolved FWHM, $\Phi_{\mathrm{d}}$, and the resolution size, $\Phi_{\mathrm{b}}$, from the Gaussian fit are in Cols. (4) and (5), respectively. The same but from the second-moment method can be found in Cols. (6) and (7). The results of direct measuremets at $10 \%$ level are given in Col. (8). Some PNe have relatively deep images and the nebular emission extends well beyond the $10 \%$ contour. In this cases we have also measured the dimensions of the $3 \sigma$ emission level above the background. The results are in Col. (9). Column (10) gives the percentage of the $3 \sigma$ level in the peak surface brightness. All the measurement results are in arcsec.

For some objects the deconvolution methods do not work well and Table 1 does not give results in these cases. It has usually happened when the nebula was extended with complex structures and/or had a strong central star or several field stars superimposed on the PN image. In these cases the numerical 
procedure fitting Gaussian or calculating moments either has failed or has given spurious results, or has just measured a star (central or one of the field stars). Note that the Gaussian fit fails more often that the second-moment method, particularly for larger nebulae.

Not all the objects published in Schwarz et al. (1992) and Górny et al. (1999) appear in Table 1. Some PNe were too faint in the images or larger than the image frame so meaningful measurements have not been possible. On the other hand, images for a few objects (those with no entry in Col. (3) in Table 1) have not been published. For most of these objects their images (although obtained with different equipement) can be found in Manchado et al. (1996).

For a few objects we had two images from independent observations. We give results from both images separately in two consecutive rows in Table 1 in these cases.

\section{Comparison of the methods}

In this section we compare the results from the three methods used to measure the PN dimensions. The aim is to study systematic effects between different methods. This will allow us to work out a procedure for deriving a consistent set of PN dimensions for all the objects, i.e. those well resolved as well as partially resolved, in our sample.

\subsection{Deconvolution methods: Gaussian versus second-moment}

As discussed above, both, Gaussian fitting and second-moment calculations have been applied to measure the FWHM of the resolution profile, $\Phi_{b}$, on our images. The results are given in Cols. (5) and (7) of Table 1. Both methods should give the same result if the instrumental profile is Gaussian. As can be seen from Table 1 this is essentially the case. The mean value and the standard deviation of the ratio $\Phi_{b}($ Gauss $) / \Phi_{b}(\mathrm{~s}-\mathrm{m})$ calculated from the data in Table 1 is $0.965 \pm 0.094$. Thus the basic assumption of the deconvolution methods, namely Gaussian resolution profile, is satisfied in our observational material.

The final results of the Gaussian and second-moment deconvolution methods are compared in Fig. 1. The figure plots the ratio of the angular diameters, $\Theta$, derived from the two methods versus the ratio of the deconvolved FWHM, $\Phi_{\mathrm{d}}$, to the resolution size, $\Phi_{\mathrm{b}}$, (i.e. parameter $\beta$ in van Hoof 2000) obtained from the Gaussian fit. The values plotted in Fig. 1 are geometric means of the values obtained from the two-dimensional measurements reported in Table 1. While deriving $\Theta$ a surface brightness distribution from a constant emissivity sphere model (shell 0.0 in van Hoof's notation) has been adopted for calculating the conversion factors. Note, however, that the diagram shown in Fig. 1 depends only weakly on the adopted surface brightness distribution.

As can be seen from Fig. 1, the two deconvolution methods do not give the same result for the bulk of objects. The second-moment method tends to give larger diameters than the Gaussian deconvolution. In the case of compact nebulae, i.e. when the Gaussian deconvolution gives $\log \Phi_{\mathrm{d}} / \Phi_{\mathrm{b}} \lesssim 0.0$, the difference becomes particularly important and increases with

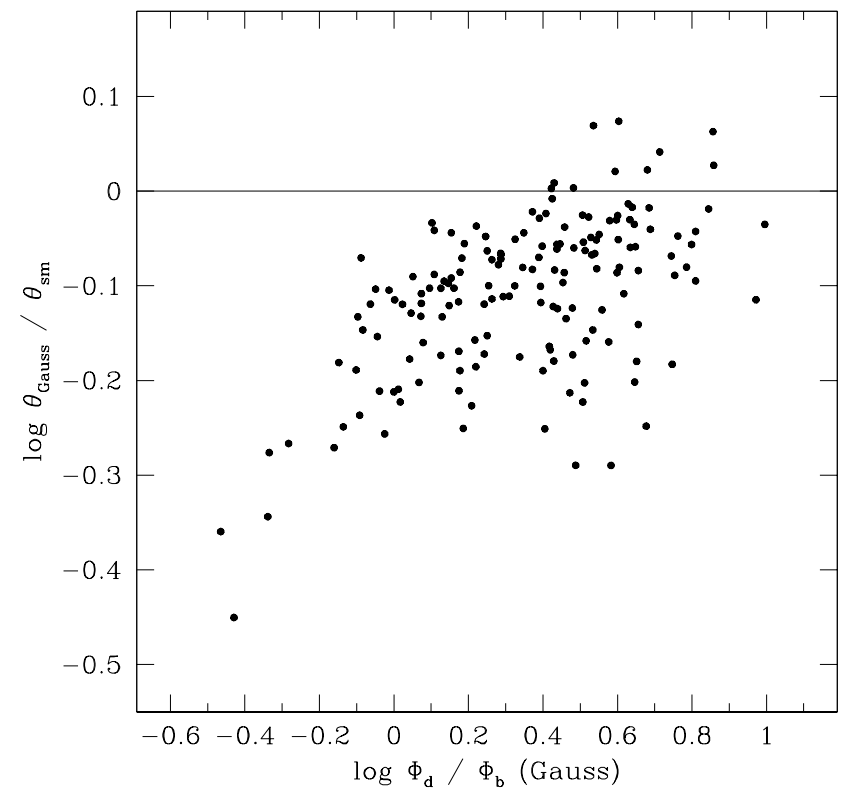

Fig. 1. Comparison of the results from the Gaussian deconvolution and the second-moment deconvolution. The ratio of the angular diameters, $\Theta$, derived from the two methods is plotted versus the ratio of the deconvolved FWHM, $\Phi_{\mathrm{d}}$, to the resolution size, $\Phi_{\mathrm{b}}$, obtained from the Gaussian fit. A constant emissivity sphere model has been adopted while deriving $\Theta$ in both methods.

decreasing PN size (as measured from the Gaussian deconvolution). Note that the vertical scatter of the points in Fig. 1, tends to become larger for more extended nebulae. On average $\log \Theta_{\text {Gauss }} / \Theta_{\mathrm{s}-\mathrm{m}}$ for objects with $\log \Phi_{\mathrm{d}} / \Phi_{\mathrm{b}} \geq 0.0$ is $-0.096 \pm 0.071$ (mean and standard deviation). When adopting a shell-like model this value would go down and for an infinitely thin shell (limit 1.0 in van Hoof's notation) it would become $-0.125 \pm 0.070$. If a constant surface brightness disc is adopted the figures would be $-0.108 \pm 0.071$.

\subsection{Gaussian deconvolution versus direct measurements at the $10 \%$ level}

Figure 2 compares the results from the Gaussian deconvolution with the $10 \%$ contour measurements. Similarly as Fig. 1, it shows the ratio of the PN diameters versus the Gaussian $\Phi_{\mathrm{d}} / \Phi_{\mathrm{b}}$ ratio. Again, the plotted values are geometric means from the measurements in Table 1. While obtaining the diameters from the Gaussian method the constant emissivity sphere model has been adopted. Note that the diagram in Fig. 2 depends on the adopted surface brightness distribution more significantly than Fig. 1 . The general pattern of the diagram remains the same but the points can shift vertically.

Figure 2 shows an increasing discrepancy between the $10 \%$ diameter and the diameter from the Gaussian deconvolution with the decreasing value of $\Phi_{\mathrm{d}} / \Phi_{\mathrm{b}}$. This is an expected effect and is due to an overestimate of the PN diameter from the direct measurements for partially resolved PNe. Note, however, that a tight correlation seen in Fig. 2 for $\log \Phi_{\mathrm{d}} / \Phi_{\mathrm{b}} \lesssim 0.0$ is partly spurious. For $\Phi$ approaching $\Phi_{\mathrm{b}}$ the $y$-axis in Fig. 2 tends to plot a reversal of the value plotted in the $x$-axis. 


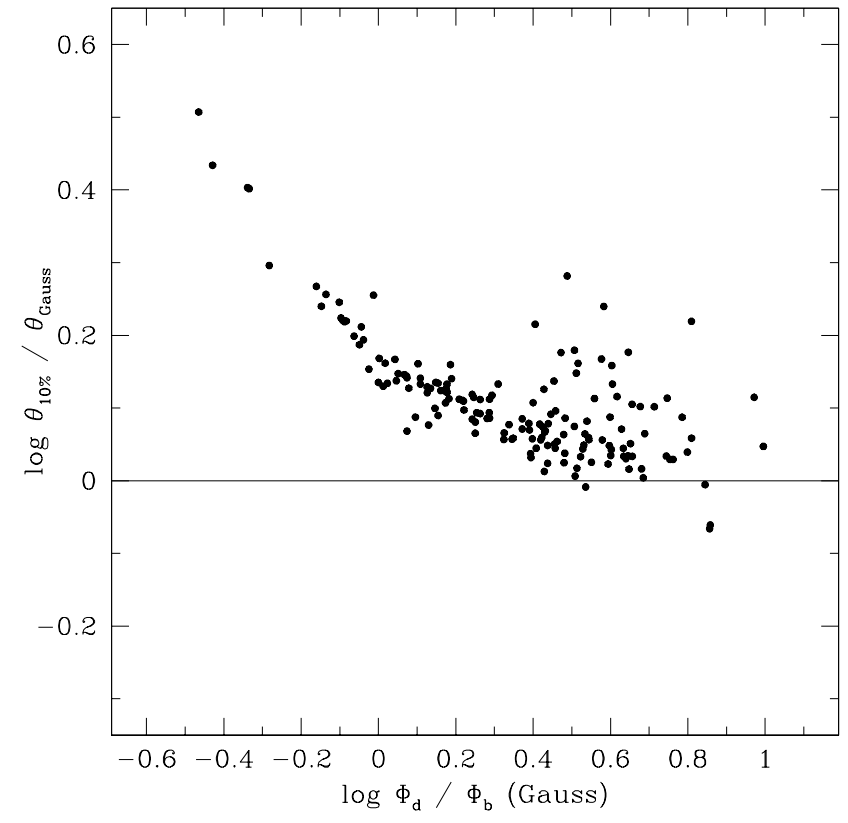

Fig. 2. Comparison of the results from the $10 \%$ contour measurements and the Gaussian deconvolution. The ratio of the angular diameters, $\Theta$, derived from the two methods is plotted versus the ratio of the deconvolved FWHM, $\Phi_{\mathrm{d}}$, to the resolution size, $\Phi_{\mathrm{b}}$, obtained from the Gaussian fit. A constant emissivity sphere model has been adopted while deriving $\Theta$ in the Gaussian deconvolution.

For small nebulae the diameters from the $10 \%$ contour would be more accurate if determined from deconvolved images. We have not attempted to apply this rather cumbersome procedure to our images. Instead we have found that a significant improvement of the $10 \%$ diameter can be obtained using a formula analogous to the standard FWHM Gaussian deconvolution (Eq. (1)) but relevant to a $10 \%$ level of the maximum, i.e.

$\Theta_{10 \%, \mathrm{~d}}=\sqrt{\Theta_{10 \%}^{2}-\left(1.823 \Phi_{\mathrm{b}}\right)^{2}}$.

We will call $\Theta_{10 \%, \mathrm{~d}}$ the deconvolved $10 \%$ diameter.

Figure 3 shows the same as Fig. 2 but using $\Theta_{10 \%, d}$ instead of $\Theta_{10 \%}$. Unlike in Fig. 2, the distribution of the points in Fig. 3 does not show any significant trend with $\Phi_{\mathrm{d}} / \Phi_{\mathrm{b}}$. This demonstrates that Eq. (2) allows to significantly improve the reliability of the PN dimension estimate from the $10 \%$ contour measurements for small, partially resolved nebulae. $\Theta_{10 \%, \mathrm{~d}}$ seems to be an estimate of compact PN diameters as reliable as that from the Gaussian deconvolution.

However, as can be seen from Fig. 3, the Gaussian deconvolution tends to underestimate the PN dimensions compared to the $10 \%$ deconvolved diameters. Note that the value of this systematic difference depends on the surface brightness distribution adopted in the Gaussian method. It is smallest for the constant emissivity sphere, i.e. for the case presented in Fig. 3, and largest for the infinitely thin shell model.

Figure 3 suggests that the two methods give the most consistent results for rather compact but not too small nebulae, i.e. having $-0.2 \lesssim \log \Phi_{\mathrm{d}} / \Phi_{\mathrm{b}} \lesssim 0.3$. The mean value of $\log \Theta_{10 \%, \mathrm{~d}} / \Theta_{\text {Gauss }}$ for these objects in Fig. 3 is $0.028 \pm 0.041$. For an infinitely thin shell (limit 1.0 in van Hoof's notation)

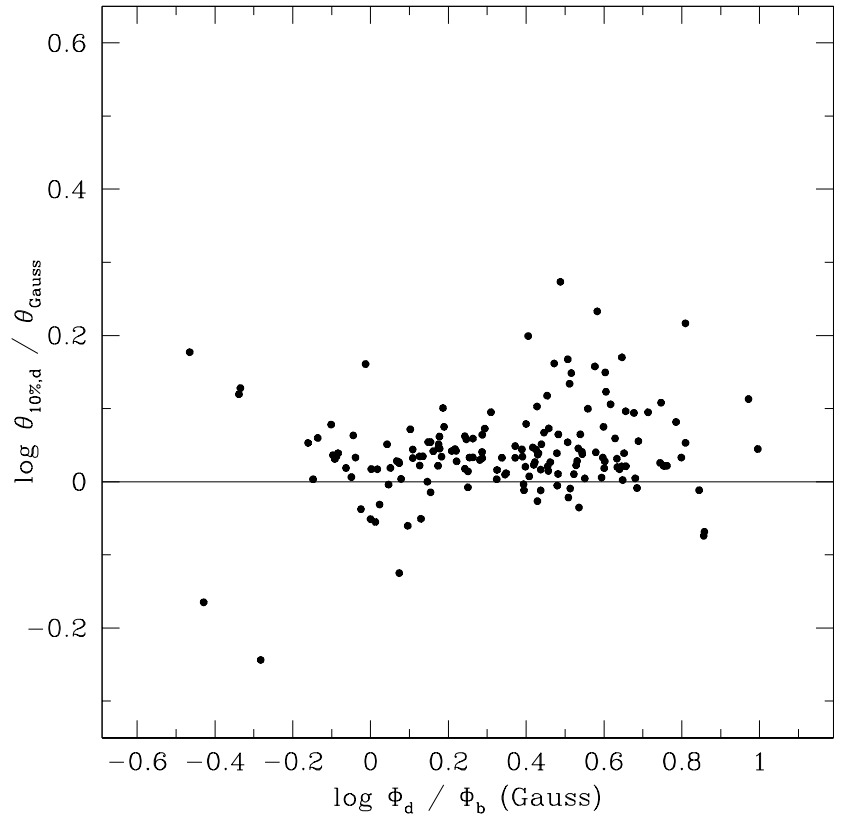

Fig. 3. The same as Fig. 2 but using the deconvolved $10 \%$ diameter, $\Theta_{10 \%, \mathrm{~d}}$, calculated from Eq. (2).

and a constant surface brightness disc the result would be $0.159 \pm 0.043$ and $0.085 \pm 0.042$, respectively.

A greater discrepancy of the results for $\log \Phi_{\mathrm{d}} / \Phi_{\mathrm{b}}>$ 0.3 can be seen from Fig. 3. The mean values of $\log \Theta_{10 \%, \mathrm{~d}} / \Theta_{\text {Gauss }}$ derived for these objects are: $0.052 \pm 0.062$ (sphere), $0.195 \pm 0.062$ (thin shell), and $0.115 \pm 0.062$ (disc). These nebulae show resolved structures on the images and their surface brightness distributions are usually far from a Gaussian. Therefore a Gaussian fitting to such objects gives uncertain, sometimes even spurious, results.

Because of low statistics, no meaningful analysis can be done for the region $\log \Phi_{\mathrm{d}} / \Phi_{\mathrm{b}}<-0.2$. A large scatter of the points is certainly due to increasing sensitivity of the results to the observational uncertainties (quadratic subtraction of two nearly equal values).

\subsection{Second-moment deconvolution versus direct measurements at $10 \%$ level}

Figures 4 and 5 compare the results from the $10 \%$ level measurements and the second-moment deconvolution. Figure 4 is analogous to Fig. 2 and uses "raw" measurements from the $10 \%$ contour. A tendency of overestimating diameters for compact PNe from the $10 \%$ measurements can be seen in Fig. 4. It is, however, much less conspicuous than in Fig. 2. The reason is that, as discussed in Sect. 5.1, for partially resolved PNe the second-moment deconvolution results in larger diameters than the Gaussian deconvolution.

The deconvolved $10 \%$ diameter, using Eq. (2) and $\Phi_{\mathrm{b}}$ from the second-moment calculations, have been applied in the diagram shown in Fig. 5. As the deconvolution of the 10\% diameter most affects the results for compact nebulae the positions of these objects are significantly shifted down in Fig. 5 comparing to Fig. 4. 


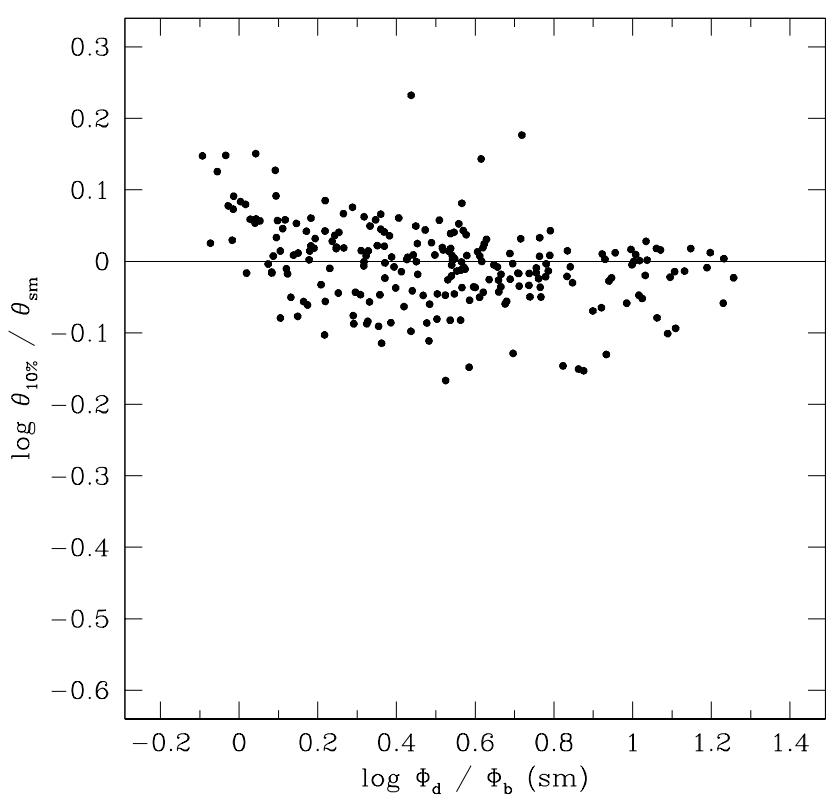

Fig. 4. Comparison of the results from the $10 \%$ contour measurements and the second-moment deconvolution. The ratio of the angular diameters, $\Theta$, derived from the two methods is plotted versus the ratio of the deconvolved FWHM, $\Phi_{\mathrm{d}}$, to the resolution size, $\Phi_{\mathrm{b}}$, obtained from the moment calculations. A constant emissivity sphere model has been adopted while deriving $\Theta$ in the second-moment deconvolution.

Figure 5 shows that the second-moment deconvolution usually overestimates the PN size compared to the $10 \%$ contour measurements. For rather large nebulae, i.e. having $\log \Phi_{\mathrm{d}} / \Phi_{\mathrm{b}} \geq 0.3$ in Fig. $5, \log \Theta_{10 \%, \mathrm{~d}} / \Theta_{\mathrm{s}-\mathrm{m}}$ is $-0.033 \pm 0.061$. The discrepancy increases for less resolved nebulae and for $\log \Phi_{\mathrm{d}} / \Phi_{\mathrm{b}}<0.3, \log \Theta_{10 \%, \mathrm{~d}} / \Theta_{\mathrm{s}-\mathrm{m}}$ is $-0.132 \pm 0.115$.

As the conversion factors in the second-moment deconvolution are independent of $\Phi_{\mathrm{d}} / \Phi_{\mathrm{b}}$ adopting the infinitely thin shell model would shift all the points in Fig. 5 upwards by factor 0.111 . For the uniform disc model the shift would be of factor 0.048 . By the same factors would respectively increase the mean values of $\log \Theta_{10 \%, \mathrm{~d}} / \Theta_{\mathrm{s}-\mathrm{m}}$ (obviously, the standard deviations would remain the same).

\subsection{Testing the methods on degraded PN images}

One of the main problems in the present study is to find which method(s) gives reliable diameter estimates for partially resolved nebulae. The analysis discussed in the previous subsections shows that the deconvolved $10 \%$ diameter and the Gaussian deconvolution give relatively consistent results down to rather poorly resolved objects, although the Gaussian deconvolution systematically underestimates the PN diameter compared to the measurements of the $10 \%$ contour. The value of this underestimate depends on the model nebula adopted in the Gaussian deconvolution. On the other hand, the secondmoment deconvolution seems to become unstable for compact objects as suggested by an increasing scatter of points in Fig. 5 for less and less resolved nebulae. However, we cannot be sure that this interpretation is correct. Nor we can say which method and when breaks to give reliable results. The reason is that we

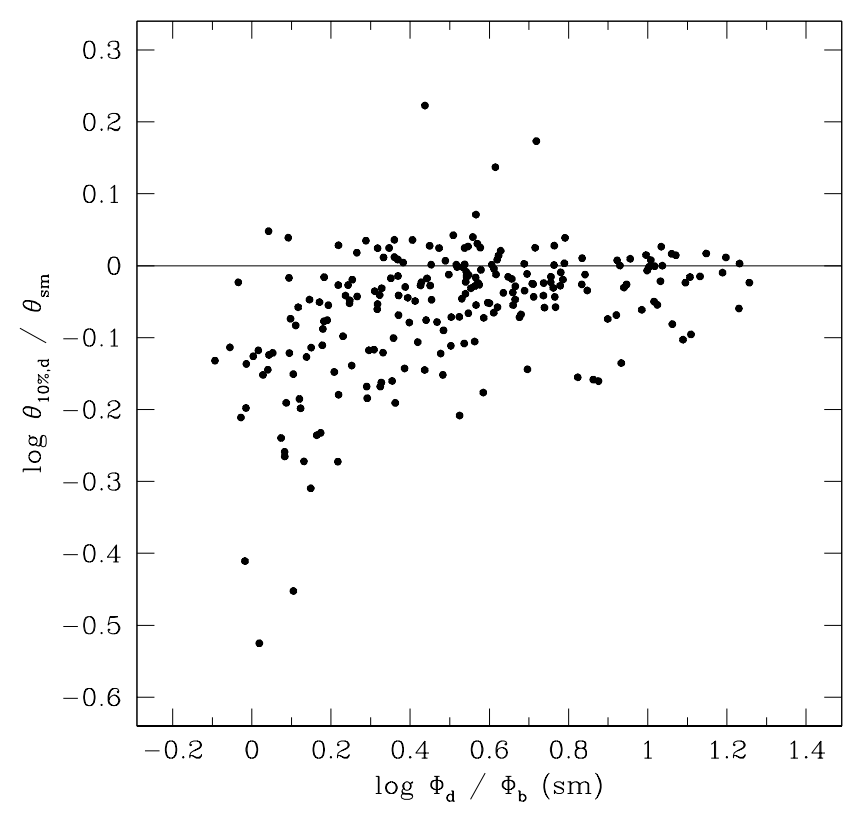

Fig. 5. The same as Fig. 4 but using the deconvolved $10 \%$ diameter, $\Theta_{10 \%, \mathrm{~d}}$, calculated from Eq. (2).

can compare only the results from different methods but we do not know what real diameters of poorly resolved nebulae are.

We have attempted to clarify the above problems by applying the methods of diameter measurements to some of our images artificially degraded to worse and worse seeing conditions, i.e. convolved with a larger and larger Gaussian profile. From the original images we know the true PN sizes so we can compare them to the results from different methods applied to less and less resolved PN images.

Although the idea is simple it could have been applied to a limited number of our images only. Obviously we have to consider rather well resolved nebulae but not too much. The object has to be several times smaller than the image size. Otherwise the image frame can impose an important cutoff when the image is convolved with a Gaussian having FWHM comparable to the PN size. The nebula has to be well exposed so its image keeps a good contrast while beeing spread over larger and larger surface. There cannot be too many bright field stars, otherwise they merge with the PN image in the convolution process. It is also desirable that among the objects investigated different morphologies are represented.

We present the results of the procedure applied to images of $5 \mathrm{PN}$, i.e. (in paranthesis usual names and morphological descriptions are given) 021.8-00.4 (M 3-28; bipolar, butterflylike), 174.2-14.6 (H3-29; ring-like but strongly asymmetric with one strong blob), 285.7-14.9 (IC 2448; round with a halo), 286.3-04.8 (NGC 3211; ring-like with two blobs), and 345.2-08.8 (Tc 1; round with an irregular halo). The selected objects have diameters between 10 and 20 arcsec. The original images have been obtained with a seeing varying from 0.5 to 2.5 arcsec. We have used our deconvolved $10 \%$ diameters from original images as true PN diameters for comparison with the results from degraded images (note that the deconvolution has introduced only minor corrections to the $10 \%$ contour measurements in these cases). The original images have been 


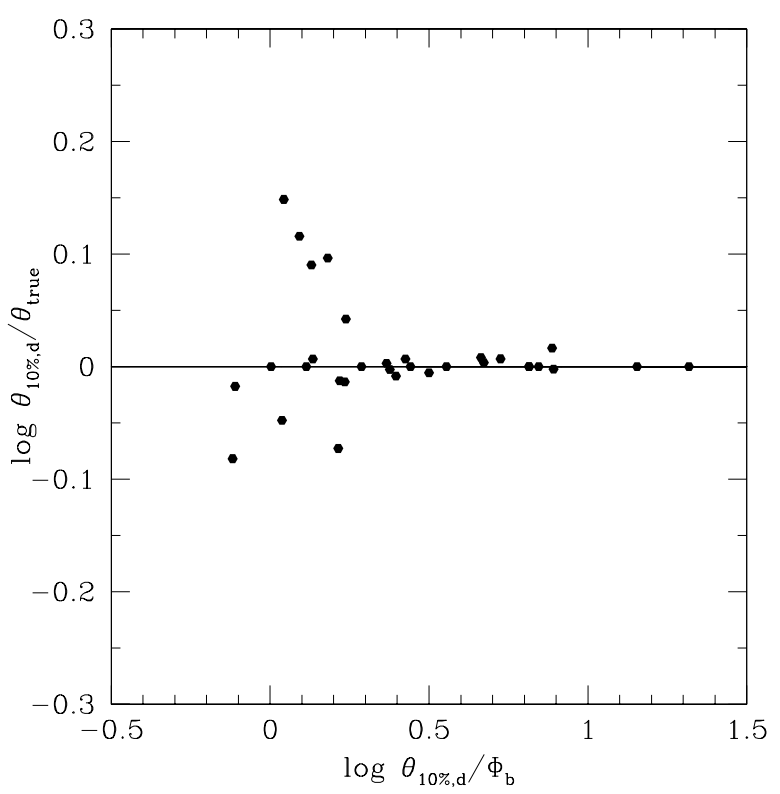

Fig. 6. Results of the $10 \%$ contour measurements on the degraded PN images. $\Theta_{10 \%, d}$ and $\Phi_{b}$ are the deconvolved $10 \%$ diameter and the seeing size, respectively, measured from the degraded images. $\Theta_{\text {true }}$ is the deconvolved $10 \%$ diameter from the original (non-degraded) images.

convolved with a Gaussian having FWHM increasing from 2.5 to 20.0 arcsec. Then our three methods have been applied to the resulting images.

We note that the results from measurements on the degraded images reproduce very well the general trends seen in Figs. 1-5.

In Fig. 6 the results of the deconvolved $10 \%$ diameter method applied to the degraded images are compared to the deconvolved $10 \%$ diameter from the original (non-degraded) images $\left(\Theta_{\text {true }}\right)$. From this figure we can conlude that the deconvolved $10 \%$ diameter measures very well the PN size down to $\log \Theta_{10 \%, \mathrm{~d}} / \Phi_{\mathrm{b}} \simeq 0.3$. This limit is practically equivalent to $\log \Phi_{\mathrm{d}}$ (Gauss) $/ \Phi_{\mathrm{b}} \simeq 0.05$. Below this value the method becomes unstable.

Results of the Gaussian deconvolution applied to the degraded images are presented in Fig. 7. A constant emissivity sphere model has been adopted. We see that for well resolved nebulae the Gaussian deconvolution underestimates the PN diameter. This is consistent with the trend seen in Fig. 3. For $\log \Phi_{\mathrm{d}} / \Phi_{\mathrm{b}} \lesssim 0.3$ the underestimate is smaller, again similar as in Fig. 3. For all the points with $\log \Phi_{\mathrm{d}} / \Phi_{\mathrm{b}}<0.3$ in Fig. 7 the mean value and the standard deviation of $\log \Theta_{\text {Gauss }} / \Theta_{\text {true }}$ are $-0.021 \pm 0.032$. This result is very close to the value $-0.028 \pm 0.041$ of $\log \Theta_{\text {Gauss }} / \Theta_{10 \%, \mathrm{~d}}$ found in Sect. 5.2 for objects having $-0.2 \lesssim \log \Phi_{\mathrm{d}} / \Phi_{\mathrm{b}} \lesssim 0.3$ in Fig. 3. For $\log \Phi_{\mathrm{d}} / \Phi_{\mathrm{b}} \lesssim-0.2$ in Fig. 7 the scatter increases which suggests that the Gaussian deconvolution becomes then unstable. Comparison of Figs. 7 and 6 seems to indicate that the Gaussian deconvolution gives somewhat more stable results than the deconvolved $10 \%$ diameter for objects having $\log \Theta_{10 \%, \mathrm{~d}} / \Phi_{\mathrm{b}} \leq 0.3$ or, equivalently,

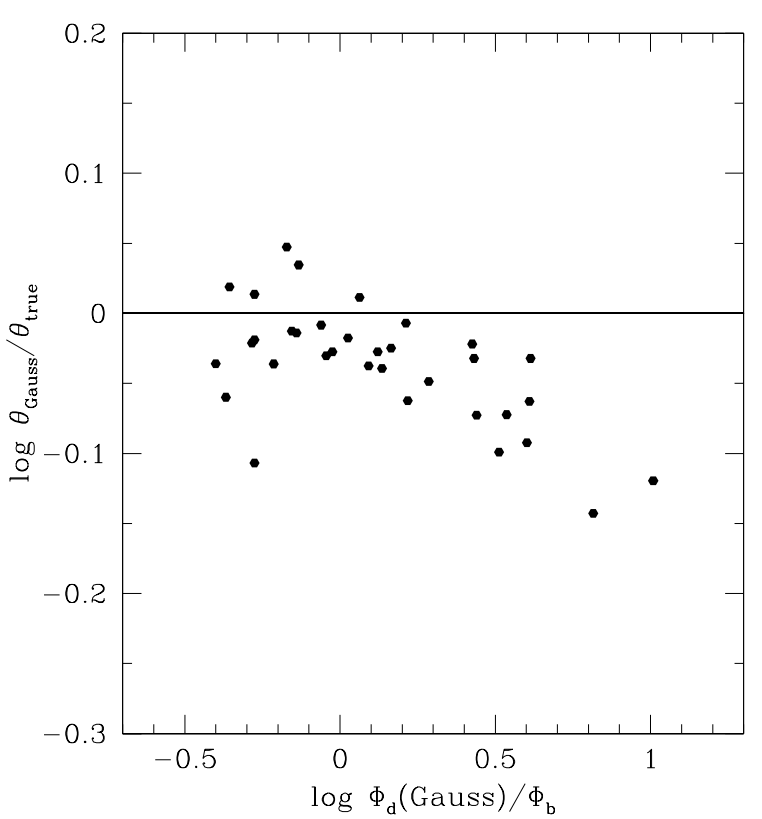

Fig. 7. The same as Fig. 6 but for the Gaussian deconvolution adopting a constant emissivity sphere model. $\Theta_{\text {true }}$ is the deconvolved $10 \%$ diameter from the original (non-degraded) images.

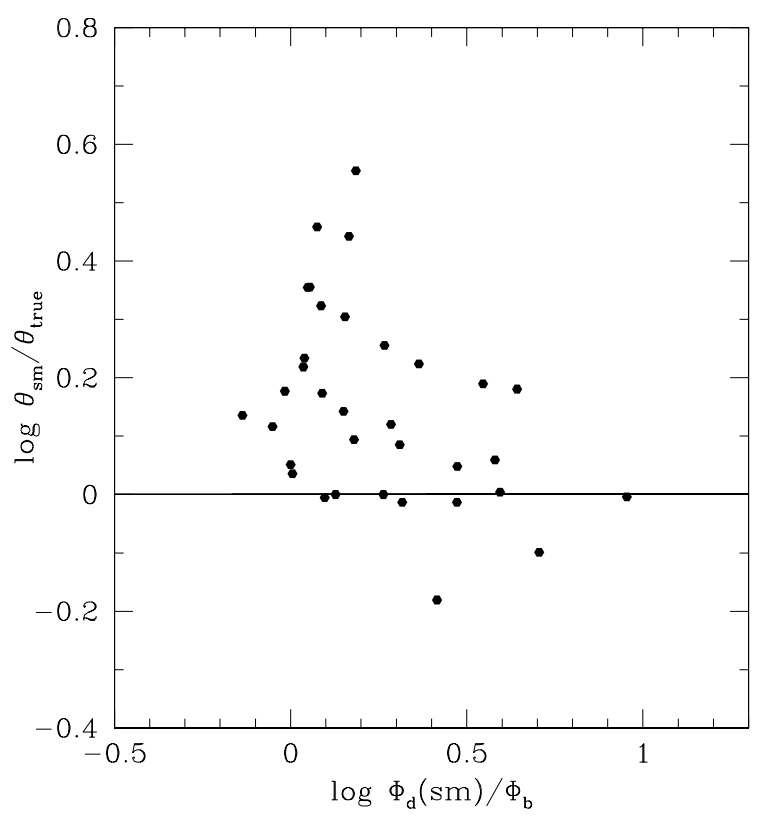

Fig. 8. The same as Fig. 6 but for the second-moment deconvolution adopting a constant emissivity sphere model. $\Theta_{\text {true }}$ is the deconvolved $10 \%$ diameter from the original (non-degraded) images.

$\log \Phi_{\mathrm{d}}$ (Gauss) $/ \Phi_{\mathrm{b}} \leq 0.05$. A better statistics would be required to clarify this point.

The results of the test of the second-moment deconvolution are presented in Fig. 8. A constant emissivity sphere model has been adopted. The figure shows that the second-moment deconvolution gives uncertain estimates of the PN diameters, especially for partially resolved nebulae $\left(\log \Phi_{\mathrm{d}} / \Phi_{\mathrm{b}}<0.3\right)$. Adopting a shell or disc model in the deconvolution would decrease the systematic difference between $\Theta_{\mathrm{s}-\mathrm{m}}$ and $\Theta_{\text {true }}$ in Fig. 8 but the uncertainity (scatter of the points) would remain 
the same. Our experience suggests that this method is sensitive to faint outer structures (halo, outer butterfly-like filaments) particularly in partially resolved nebulae.

\section{PN diameters}

One of the primary goals of our work is to obtain a homogeneous set of PN dimensions, a set of results which have, as far as possible, the same physical meaning for all the objects in our sample. As a primary method for deriving the PN dimensions we have adopted the direct measurements at the $10 \%$ level of the peak surface brightness. This seems to be the only reasonable method for well resolved nebulae. In order to account for the finite resolution in the case of smaller objects we have used the deconvolved $10 \%$ diameters, $\Theta_{10 \%, \mathrm{~d}}$, instead of $\Theta_{10 \%}$. As discussed in Sect. $5.4 \Theta_{10 \%, \mathrm{~d}}$ is reliable down to $\log \Theta_{10 \%, \mathrm{~d}} / \Phi_{\mathrm{b}} \simeq 0.3$. For the smallest objects when results from any method become more and more uncertain we have used $\Theta_{10 \%, \mathrm{~d}}$ and the results from the Gaussian deconvolution. Following discussion in Sect. 5.4 we have prefered not to use the results from the second-moment deconvolution. In more detail our prescription is as follows.

For all the objects with $\log \Theta_{10 \%, \mathrm{~d}} / \Phi_{\mathrm{b}} \geq 0.3$ we have adopted $\Theta_{10 \%, \mathrm{~d}}$. We have used $\Phi_{\mathrm{b}}$ from Gaussian fit. For more compact objects, i.e. when $\log \Theta_{10 \%, \mathrm{~d}} / \Phi_{\mathrm{b}}<0.3$ we have adopted a mean value from $\Theta_{10 \%, \mathrm{~d}}$ and $\Theta$ resulting from the Gaussian deconvolution adopting the constant emissivity sphere model. In order to correct for the systematic underestimate of the Gaussian method, as discussed in Sects. 5.2 and 5.4, the Gaussian $\Theta$ have been multiplied by factor 1.06 before deriving the mean value.

The results are presented in Table 2. Columns 1 and 2 give the PNG numbers and the usual names, respectively. The diameters derived according to the above prescription are given in Col. 3. In the case of partially resolved objects having $\log \Theta_{10 \%, \mathrm{~d}} / \Phi_{\mathrm{b}}<0.3$ only one value is given which is the geometric mean from the two dimension measurements. A colon indicates compact objects for which the Gaussian deconvolution resulted in a diameter different by more than $30 \%$ from the deconvolved $10 \%$ diameter. Objects practically unresolved in our images are marked with "stellar". For a few objects having two independent measurements in Table 1 mean values derived from them are given in Table 2 .

We have compared the results from Table 2 with the diameters from our data base resulting from a compilation of the data published in the literature. This data base has originally been made for purposes of the Strasbourg-ESO catalogue (Acker et al. 1992). Its updated version has been described and used in Siódmiak \& Tylenda (2001). The results of the comparison are shown in Fig. 9. In most cases the agreement is quite good although our measurements tend to give somewhat larger diameters than the values adopted from the literature. The mean value of the ratio of our diameters (from Table 2) to the compiled diameters is 1.24 with a standard deviation of 0.71 . In some cases, however, the discrepancy is significant. For small nebulae a large scatter of the points in Fig. 9 is not surprising. Any measurement, VLA or optical, of a partially resolved nebulae gives an uncertain result. Significant discrepancies in
Table 2. Nebular diameters (in arcsec).

\begin{tabular}{|c|c|c|c|}
\hline $\begin{array}{l}\text { PN G } \\
\text { (1) }\end{array}$ & $\begin{array}{c}\text { Main Name } \\
\text { (2) }\end{array}$ & \multicolumn{2}{|c|}{$\begin{array}{c}\text { diameter } \\
\text { (3) }\end{array}$} \\
\hline $000.1+17.2$ & PC 12 & $2.3 \mathrm{x}$ & 2.2 \\
\hline 000.1-01.1 & M 3-43 & $4.1 \mathrm{x}$ & 2.6 \\
\hline $000.1-05.6$ & H 2-40 & $18.3 \mathrm{x}$ & 16.9 \\
\hline $000.2-01.9$ & M 2-19 & $9.4 \mathrm{x}$ & 8.5 \\
\hline $000.3+12.2$ & IC 4634 & $20.5 \mathrm{x}$ & 6.6 \\
\hline 000.4-01.9 & M 2-20 & $4.1 \mathrm{x}$ & 3.4 \\
\hline $000.7+04.7$ & H 2-11 & 2.0 & \\
\hline $001.0+01.9$ & K 1- 4 & $48.1 \mathrm{x}$ & 33.6 \\
\hline $001.5-06.7$ & SwSt 1 & $5.6 \mathrm{x}$ & 5.2 \\
\hline 002.0-13.4 & IC 4776 & $8.5 \mathrm{x}$ & 4.0 \\
\hline $002.2-06.3$ & H 1-63 & $3.8 \mathrm{x}$ & 3.2 \\
\hline $002.2-09.4$ & Cn 1-5 & $7.4 \mathrm{x}$ & 7.4 \\
\hline $002.4+05.8$ & NGC 6369 & $33.0 \mathrm{x}$ & 32.7 \\
\hline 002.5-01.7 & Pe 2-11 & $7.8 \mathrm{x}$ & 6.5 \\
\hline $002.7-04.8$ & M 1-42 & $13.1 \mathrm{x}$ & 11.3 \\
\hline $002.7-52.4$ & IC $5148-50$ & $132.5 \mathrm{x}$ & 127.8 \\
\hline $002.8+01.7$ & H 2-20 & stellar & \\
\hline $003.1+02.9$ & $\mathrm{Hb} 4$ & $11.1 \mathrm{x}$ & 6.8 \\
\hline $003.1+03.4$ & H 2-17 & $4.8 \mathrm{x}$ & 3.9 \\
\hline $003.5-02.4$ & IC 4673 & $22.0 \mathrm{x}$ & 15.2 \\
\hline $003.5-04.6$ & NGC 6565 & $10.8 \mathrm{x}$ & 10.5 \\
\hline 003.6-02.3 & M 2-26 & $10.5 \mathrm{x}$ & 10.4 \\
\hline $003.7+07.9$ & H 2- 8 & $11.9 \mathrm{x}$ & 6.7 \\
\hline $003.8-04.5$ & H 2-41 & $9.3 \mathrm{x}$ & 9.2 \\
\hline 003.8-17.1 & $\mathrm{Hb} 8$ & $0.8:$ & \\
\hline 003.9-14.9 & $\mathrm{Hb} 7$ & 3.5 & \\
\hline 004.0-11.1 & M 3-29 & $9.7 \mathrm{x}$ & 8.6 \\
\hline $004.8-22.7$ & He 2-436 & $3.6 \mathrm{x}$ & 2.8 \\
\hline 004.9-04.9 & M 1-44 & $6.0 \mathrm{x}$ & 5.4 \\
\hline $005.0+03.0$ & Pe 1- 9 & $13.6 \mathrm{x}$ & 13.4 \\
\hline 005.0-03.9 & H 2-42 & $13.0 \mathrm{x}$ & 11.9 \\
\hline 005.1-08.9 & Hf $2-2$ & $21.7 \mathrm{x}$ & 21.7 \\
\hline $006.0+03.1$ & M 1-28 & $33.1 \mathrm{x}$ & 30.3 \\
\hline $006.1+08.3$ & M 1-20 & $2.5 \mathrm{x}$ & 2.3 \\
\hline 006.8-08.6 & Al 1 & $15.00 \mathrm{x}$ & 12.3 \\
\hline $007.2+01.8$ & $\mathrm{Hb} 6$ & $7.7 \mathrm{x}$ & 6.8 \\
\hline $008.0+03.9$ & NGC 6445 & $45.3 \mathrm{x}$ & 36.2 \\
\hline 008.3-01.1 & M 1-40 & $9.2 \mathrm{x}$ & 7.5 \\
\hline 008.3-07.3 & NGC 6644 & $4.4 \mathrm{x}$ & 4.3 \\
\hline 009.4-05.0 & NGC 6629 & $16.6 \mathrm{x}$ & 15.5 \\
\hline $009.4-09.8$ & M 3-32 & $8.1 \mathrm{x}$ & 6.8 \\
\hline $009.6+10.5$ & A 41 & $20.2 \mathrm{x}$ & 17.3 \\
\hline $009.6+14.8$ & NGC 6309 & $22.8 \mathrm{x}$ & 12.4 \\
\hline 009.6-10.6 & M 3-33 & $7.4 \mathrm{x}$ & 7.3 \\
\hline 010.7-06.4 & IC 4732 & $1.4:$ & \\
\hline $010.8+18.0$ & M 2- 9 & $37.0 \times$ & 11.0 \\
\hline 010.8-01.8 & NGC 6578 & $12.1 \mathrm{x}$ & 11.8 \\
\hline 011.0-05.1 & M 1-47 & $6.2 \mathrm{x}$ & 5.3 \\
\hline $011.1+11.5$ & M 2-13 & $3.0 \mathrm{x}$ & 2.4 \\
\hline 011.7-00.6 & NGC 6567 & $8.1 \mathrm{x}$ & 6.4 \\
\hline $011.9+04.2$ & M 1-32 & $9.1 \mathrm{x}$ & 8.0 \\
\hline $012.5-09.8$ & M 1-62 & $4.8 \mathrm{x}$ & 4.6 \\
\hline 013.0-04.3 & Pe 2-14 & $5.5 \mathrm{x}$ & 5.3 \\
\hline $013.3+32.7$ & Sn 1 & $5.9 \mathrm{x}$ & 5.0 \\
\hline 014.6-04.3 & M 1-50 & $4.2 \mathrm{x}$ & 3.9 \\
\hline 016.4-01.9 & M 1-46 & $12.1 \mathrm{x}$ & 11.3 \\
\hline 017.6-10.2 & A 51 & $59.2 \times$ & 59.0 \\
\hline 017.9-04.8 & M 3-30 & $19.1 \mathrm{x}$ & 18.4 \\
\hline
\end{tabular}


Table 2. continued.

\begin{tabular}{|c|c|c|c|}
\hline (1) & (2) & \multicolumn{2}{|l|}{ (3) } \\
\hline $020.9-01.1$ & M 1-51 & $15.4 \mathrm{x}$ & 8.3 \\
\hline $021.2-03.9$ & We 1-7 & $20.5 \mathrm{x}$ & 19.7 \\
\hline $021.7-00.6$ & M 3-55 & $12.2 \mathrm{x}$ & 9.3 \\
\hline $021.8-00.4$ & M 3-28 & $24.1 \mathrm{x}$ & 12.1 \\
\hline $023.9-02.3$ & M 1-59 & $6.7 \mathrm{x}$ & 6.0 \\
\hline $024.2+05.9$ & M 4- 9 & $47.9 \mathrm{x}$ & 42.6 \\
\hline $024.3-03.3$ & Pe $1-17$ & $14.7 \mathrm{x}$ & 7.6 \\
\hline 025.8-17.9 & NGC 6818 & $24.7 x$ & 24.7 \\
\hline 025.9-10.9 & $\mathrm{Na} 2$ & $6.3 \mathrm{x}$ & 5.7 \\
\hline 027.3-03.4 & A 49 & $54.1 \mathrm{x}$ & 38.2 \\
\hline $027.7+00.7$ & M 2-45 & $9.4 \mathrm{x}$ & 7.6 \\
\hline $028.0+10.2$ & WeSb 3 & $50.7 \mathrm{x}$ & 43.1 \\
\hline 028.7-03.9 & Pe 1-21 & $11.1 \mathrm{x}$ & 10.4 \\
\hline $029.2-05.9$ & NGC 6751 & $24.1 \times$ & 23.2 \\
\hline $030.8+03.4$ & A 47 & $17.5 \mathrm{x}$ & 12.3 \\
\hline $031.0-10.8$ & M 3-34 & $7.4 \mathrm{x}$ & 6.4 \\
\hline 033.0-05.3 & A 55 & $56.8 \mathrm{x}$ & 52.3 \\
\hline 033.1-06.3 & NGC 6772 & $80.7 \mathrm{x}$ & 70.8 \\
\hline 033.8-02.6 & NGC 6741 & $9.1 \mathrm{x}$ & 6.5 \\
\hline $034.5-06.7$ & NGC 6778 & $21.4 \mathrm{x}$ & 15.5 \\
\hline 035.9-01.1 & Sh 2- 71 & $132.4 \mathrm{x}$ & 74.9 \\
\hline 037.5-05.1 & A 58 & $44.7 x$ & 33.7 \\
\hline $037.7-34.5$ & NGC 7009 & $28.0 \mathrm{x}$ & 22.0 \\
\hline $037.8-06.3$ & NGC 6790 & $4.4 \mathrm{x}$ & 3.4 \\
\hline $038.1-25.4$ & A 70 & $45.2 \mathrm{x}$ & 37.8 \\
\hline $038.2+12.0$ & Cn 3-1 & $5.7 \mathrm{x}$ & 4.6 \\
\hline 039.5-02.7 & M 2-47 & $6.9 \mathrm{x}$ & 4.9 \\
\hline 040.3-00.4 & A 53 & $31.9 \mathrm{x}$ & 31.1 \\
\hline 042.9-06.9 & NGC 6807 & $2.0 \mathrm{x}$ & 1.9 \\
\hline $043.1+03.8$ & M 1-65 & $4.2 \mathrm{x}$ & 4.0 \\
\hline $043.3+11.6$ & M 3-27 & $2.8 \mathrm{x}$ & 2.3 \\
\hline $045.4-02.7$ & Vy 2-2 & $3.1 \mathrm{x}$ & 2.6 \\
\hline 045.7-04.5 & NGC 6804 & $58.3 \times$ & 48.6 \\
\hline 046.4-04.1 & NGC 6803 & $5.4 \mathrm{x}$ & 5.1 \\
\hline 054.1-12.1 & NGC 6891 & $13.5 \mathrm{x}$ & 12.7 \\
\hline 061.4-09.5 & NGC 6905 & $43.3 \mathrm{x}$ & 35.6 \\
\hline $062.4-00.2$ & M 2-48 & $19.4 \mathrm{x}$ & 11.3 \\
\hline $065.2-05.6$ & He $1-6$ & $40.5 \mathrm{x}$ & 21.5 \\
\hline $066.7-28.2$ & NGC 7094 & $102.5 \mathrm{x}$ & 99.4 \\
\hline $069.2+03.8$ & K 3-46 & $36.2 \mathrm{x}$ & 23.5 \\
\hline 069.4-02.6 & NGC 6894 & $56.4 \mathrm{x}$ & 53.3 \\
\hline $118.8-74.7$ & NGC 246 & $260.4 x$ & 226.7 \\
\hline $161.2-14.8$ & IC 2003 & $10.0 \mathrm{x}$ & 8.1 \\
\hline $171.3-25.8$ & Ba 1 & $39.8 x$ & 38.3 \\
\hline $174.2-14.6$ & Н 3-29 & $23.8 x$ & 18.8 \\
\hline $189.1+19.8$ & NGC 2371-72 & $48.9 \mathrm{x}$ & 30.6 \\
\hline $190.3-17.7$ & J 320 & $9.4 \mathrm{x}$ & 6.4 \\
\hline $194.2+02.5$ & J 900 & $8.2 \mathrm{x}$ & 7.8 \\
\hline 196.6-10.9 & NGC 2022 & $27.9 x$ & 25.5 \\
\hline $197.2-14.2$ & K 1- 7 & $37.2 \mathrm{x}$ & 36.0 \\
\hline 198.6-06.3 & A 12 & $44.1 \mathrm{x}$ & 38.5 \\
\hline 201.9-04.6 & We 1-4 & $41.4 \mathrm{x}$ & 37.6 \\
\hline 206.4-40.5 & NGC 1535 & $33.3 \mathrm{x}$ & 32.1 \\
\hline $212.0+04.3$ & M 1- 9 & 2.7 & \\
\hline $214.9+07.8$ & A 20 & $67.3 x$ & 60.5 \\
\hline 216.3-04.4 & We $1-5$ & $20.3 x$ & 19.3 \\
\hline 221.3-12.3 & IC 2165 & $9.3 \mathrm{x}$ & 8.9 \\
\hline $221.7+05.3$ & M 3- 3 & $16.6 \mathrm{x}$ & 15.8 \\
\hline $224.9+01.0$ & We 1-6 & $60.9 x$ & 58.4 \\
\hline $226.4-03.7$ & PB 1 & $10.6 \mathrm{x}$ & 9.5 \\
\hline
\end{tabular}

Table 2. continued.

\begin{tabular}{|c|c|c|}
\hline$(1)$ & (2) & (3) \\
\hline $226.7+05.6$ & M 1-16 & $7.7 \times \quad 5.5$ \\
\hline $228.8+05.3$ & M 1-17 & 3.8 \\
\hline 229.6-02.7 & K 1-10 & $51.3 \times 46.0$ \\
\hline $231.4+04.3$ & M 1-18 & $34.9 \times 32.9$ \\
\hline $231.8+04.1$ & NGC 2438 & $80.7 \times 78.3$ \\
\hline $232.4-01.8$ & M 1-13 & $18.6 \times 11.6$ \\
\hline $232.8-04.7$ & M 1-11 & $5.2 \times 5.1$ \\
\hline $233.5-16.3$ & A 15 & $36.6 \times 34.7$ \\
\hline $234.8+02.4$ & NGC 2440 & $58.9 \times 25.1$ \\
\hline $234.9-01.4$ & M 1-14 & $5.7 \times \quad 5.2$ \\
\hline $236.7+03.5$ & K 1-12 & $44.1 \times 36.4$ \\
\hline $238.9+07.3$ & Sa 2-21 & $40.3 \times 34.4$ \\
\hline $239.6+13.9$ & NGC 2610 & $49.7 \times 47.6$ \\
\hline $240.3-07.6$ & M 3- 2 & $12.3 \times 9.1$ \\
\hline $242.6-11.6$ & M 3- 1 & $12.6 \times 10.8$ \\
\hline $243.3-01.0$ & NGC 2452 & $18.3 \times 12.4$ \\
\hline $245.4+01.6$ & M 3- 5 & $8.3 \times 7.3$ \\
\hline $248.8-08.5$ & M 4- 2 & $8.2 \times 7.1$ \\
\hline $249.0+06.9$ & SaSt $1-1$ & 1.2 \\
\hline $250.3+00.1$ & A 26 & $37.5 \times 36.7$ \\
\hline $252.6+04.4$ & K 1- 1 & $51.3 \times 47.5$ \\
\hline $258.1-00.3$ & He 2- 9 & $5.9 \times 4.7$ \\
\hline $259.1+00.9$ & He 2- 11 & $121.7 \times 64.0$ \\
\hline $261.0+32.0$ & NGC 3242 & $31.5 \times 30.7$ \\
\hline $261.9+08.5$ & NGC 2818 & $56.2 \times 46.0$ \\
\hline $262.6-04.6$ & Wray $17-18$ & $17.2 \times 16.8$ \\
\hline $264.4-12.7$ & He 2- 5 & $3.8 \times 3.6$ \\
\hline $265.7+04.1$ & NGC 2792 & $17.9 \times 16.4$ \\
\hline $268.4+02.4$ & PB 5 & $1.7 \times \quad 1.6$ \\
\hline $272.1+12.3$ & NGC 3132 & $59.9 \times 45.6$ \\
\hline $273.2-03.7$ & He 2- 18 & $16.4 \times 13.7$ \\
\hline $274.3+09.1$ & Lo 4 & $41.6 \times 38.9$ \\
\hline $274.6+02.1$ & He $2-35$ & $4.0 \times 3.6$ \\
\hline $274.6+03.5$ & He 2- 37 & $26.1 \times 22.1$ \\
\hline $275.0-04.1$ & PB 4 & $12.2 \times 10.2$ \\
\hline $275.2-02.9$ & He 2- 28 & $10.8 \times 10.0$ \\
\hline $275.2-03.7$ & He $2-25$ & $54.2 \times 10.6$ \\
\hline 275.8-02.9 & He 2- 29 & $16.0 \times 11.8$ \\
\hline 277.1-03.8 & NGC 2899 & $68.5 \times 59.8$ \\
\hline 278.1-05.9 & NGC 2867 & $14.4 \times 13.9$ \\
\hline 279.6-03.1 & He 2- 36 & $24.8 \times 15.3$ \\
\hline $283.8+02.2$ & Мy 60 & $10.1 \times 10.1$ \\
\hline $283.8-04.2$ & He 2- 39 & $12.4 \times 12.2$ \\
\hline 283.9-01.8 & Hf 4 & $29.1 \times 21.0$ \\
\hline $285.4+02.2$ & Pe 2- 7 & $5.6 \times 4.4$ \\
\hline $285.4-05.3$ & IC 2553 & $11.5 \times 7.4$ \\
\hline $285.6-02.7$ & He 2- 47 & $4.9 \times 4.4$ \\
\hline $285.7+01.2$ & Pe 1- 2 & $4.0 \times 3.1$ \\
\hline 285.7-14.9 & IC 2448 & $10.7 \times 10.0$ \\
\hline $286.3-04.8$ & NGC 3211 & $16.1 \times 15.9$ \\
\hline 288.4-02.4 & Pe 1- 3 & $10.9 \times 8.8$ \\
\hline $289.8+07.7$ & He $2-63$ & 3.0 \\
\hline $291.4+19.2$ & ESO $320-28$ & $30.4 \times 27.2$ \\
\hline $291.6-04.8$ & IC 2621 & $4.0 \times 3.6$ \\
\hline $291.7+03.7$ & He 2- 64 & $9.1 \times 8.3$ \\
\hline $292.4+04.1$ & PB 8 & $6.6 \times 6.5$ \\
\hline $292.6+01.2$ & NGC 3699 & $47.0 \times 37.0$ \\
\hline $292.8+01.1$ & He 2- 67 & $5.2 \times 2.8$ \\
\hline $293.6+01.2$ & He 2- 70 & $34.6 \times 13.6$ \\
\hline $294.1+14.4$ & Lo 6 & $77.0 \times 74.4$ \\
\hline
\end{tabular}


Table 2. continued.

\begin{tabular}{|c|c|c|c|}
\hline (1) & (2) & (3) & \\
\hline $294.6+04.7$ & NGC 3918 & $18.7 \mathrm{x}$ & 17.1 \\
\hline $294.9-04.3$ & He 2- 68 & 2.5 & \\
\hline 296.3-03.0 & He 2- 73 & $3.3 \mathrm{x}$ & 2.5 \\
\hline $296.6-20.0$ & NGC 3195 & $39.5 \times$ & 33.8 \\
\hline $297.4+03.7$ & He 2- 78 & 3.5 & \\
\hline $298.1-00.7$ & He 2- 77 & $25.4 \mathrm{x}$ & 14.1 \\
\hline $298.2-01.7$ & He 2- 76 & $17.0 \mathrm{x}$ & 17.0 \\
\hline 298.3-04.8 & NGC 4071 & $72.4 x$ & 52.7 \\
\hline $299.0+18.4$ & K 1-23 & $64.3 x$ & 56.4 \\
\hline $299.5+02.4$ & He 2- 82 & $31.8 \mathrm{x}$ & 25.4 \\
\hline $299.8-01.3$ & He 2- 81 & $7.3 \mathrm{x}$ & 6.5 \\
\hline $300.2+00.6$ & He 2- 83 & $4.7 \mathrm{x}$ & 4.5 \\
\hline $300.4-00.9$ & He 2- 84 & $35.8 \mathrm{x}$ & 23.7 \\
\hline $300.5-01.1$ & He 2- 85 & $9.2 \mathrm{x}$ & 7.9 \\
\hline $300.7-02.0$ & He 2- 86 & 3.2 & \\
\hline $304.5-04.8$ & IC 4191 & $5.3 \mathrm{x}$ & 4.5 \\
\hline $304.8+05.1$ & He 2- 88 & 1.7 & \\
\hline $305.1+01.4$ & He 2- 90 & $3.2 \mathrm{x}$ & 3.1 \\
\hline $306.4-00.6$ & Th 2- A & $27.3 \mathrm{x}$ & 24.8 \\
\hline $307.2-03.4$ & NGC 5189 & $163.4 \times$ & 108.2 \\
\hline $307.2-09.0$ & He 2- 97 & 2.3 & \\
\hline $307.5-04.9$ & MyCn 18 & $17.3 \mathrm{x}$ & 9.8 \\
\hline $308.6-12.2$ & He 2-105 & $41.5 \mathrm{x}$ & 40.7 \\
\hline $309.0+00.8$ & He 2- 96 & 2.8 & \\
\hline $309.0-04.2$ & He 2- 99 & $27.9 \mathrm{x}$ & 23.4 \\
\hline $309.1-04.3$ & NGC 5315 & $10.7 \mathrm{x}$ & 9.2 \\
\hline $310.7-02.9$ & He $2-103$ & $22.1 \mathrm{x}$ & 20.9 \\
\hline $311.0+02.4$ & SuWt 2 & $86.5 \mathrm{x}$ & 43.4 \\
\hline $311.4+02.8$ & He 2-102 & $11.7 \mathrm{x}$ & 11.3 \\
\hline $312.3+10.5$ & NGC 5307 & $18.8 \mathrm{x}$ & 12.9 \\
\hline $312.6-01.8$ & He 2-107 & $10.7 \mathrm{x}$ & 8.3 \\
\hline $315.0-00.3$ & He $2-111$ & $29.4 \mathrm{x}$ & 14.5 \\
\hline $315.1-13.0$ & He $2-131$ & $10.0 \mathrm{x}$ & 9.6 \\
\hline $315.4+05.2$ & He 2-109 & $11.0 \mathrm{x}$ & 7.5 \\
\hline $315.4+09.4$ & He 2-104 & $85.6 \mathrm{x}$ & 34.4 \\
\hline $315.7+05.5$ & LoTr 8 & $28.4 \mathrm{x}$ & 25.1 \\
\hline $316.1+08.4$ & He 2-108 & $13.6 \mathrm{x}$ & 12.3 \\
\hline $317.1-05.7$ & He 2-119 & $63.3 x$ & 60.4 \\
\hline $318.3-02.0$ & He $2-114$ & $26.1 \mathrm{x}$ & 21.4 \\
\hline $318.3-02.5$ & He 2-116 & $47.9 x$ & 46.7 \\
\hline $319.2+06.8$ & He $2-112$ & $6.9 \mathrm{x}$ & 6.3 \\
\hline $319.6+15.7$ & IC 4406 & $46.4 \mathrm{x}$ & 29.9 \\
\hline $320.1-09.6$ & He $2-138$ & $6.7 x$ & 6.0 \\
\hline $320.3-28.8$ & He 2-434 & $7.4 \mathrm{x}$ & 5.1 \\
\hline $320.9+02.0$ & He 2-117 & $5.4 \mathrm{x}$ & 4.4 \\
\hline $321.3+02.8$ & He $2-115$ & $3.4 \mathrm{x}$ & 2.4 \\
\hline $321.3-16.7$ & He 2-185 & $2.9 \mathrm{x}$ & 2.5 \\
\hline $321.8+01.9$ & He 2-120 & $36.1 \mathrm{x}$ & 26.5 \\
\hline $322.1-06.6$ & He 2-136 & $7.3 \mathrm{x}$ & 4.8 \\
\hline $322.4-00.1$ & Pe 2- 8 & 2.5 & \\
\hline $322.4-02.6$ & Mz 1 & $49.3 \mathrm{x}$ & 35.3 \\
\hline $322.5-05.2$ & NGC 5979 & $20.2 \mathrm{x}$ & 19.1 \\
\hline $323.1-02.5$ & He 2-132 & $20.8 x$ & 18.9 \\
\hline $323.9+02.4$ & He $2-123$ & $6.9 \mathrm{x}$ & 6.6 \\
\hline $324.2+02.5$ & He $2-125$ & $3.8 \mathrm{x}$ & 2.9 \\
\hline $324.8-01.1$ & He $2-133$ & 1.8 & \\
\hline $325.0+03.2$ & He 2-129 & 2.9 & \\
\hline $325.4-04.0$ & He 2-141 & $13.0 \mathrm{x}$ & 10.8 \\
\hline $325.8+04.5$ & He $2-128$ & $1.7:$ & \\
\hline $325.8-12.8$ & He $2-182$ & $3.1 \mathrm{x}$ & 2.8 \\
\hline
\end{tabular}

Table 2. continued.

\begin{tabular}{|c|c|c|}
\hline$(1)$ & (2) & (3) \\
\hline $326.0-06.5$ & He 2-151 & $1.8 \times 1.7$ \\
\hline $327.1-01.8$ & He $2-140$ & 4.1 \\
\hline $327.1-02.2$ & He $2-142$ & $4.2 \times 3.1$ \\
\hline $327.5+13.3$ & He 2-118 & 1.3 \\
\hline $327.8+10.0$ & NGC 5882 & $15.6 \times 12.9$ \\
\hline $327.8-01.6$ & He 2-143 & 3.7 \\
\hline $327.8-06.1$ & He $2-158$ & 2.0 \\
\hline $327.8-07.2$ & He $2-163$ & $22.1 \times 21.8$ \\
\hline $327.9-04.3$ & He 2-147 & $4.8 \times \quad 3.7$ \\
\hline $328.2+01.3$ & Lo 10 & $28.1 \times 25.2$ \\
\hline $328.9-02.4$ & He $2-146$ & $38.4 \times 30.5$ \\
\hline $330.6-02.1$ & He $2-153$ & $18.9 \times 13.1$ \\
\hline $330.6-03.6$ & He 2-159 & $15.2 \times 10.5$ \\
\hline $330.7+04.1$ & Cn 1-1 & stellar \\
\hline $331.3+16.8$ & NGC 5873 & $7.1 \times 5.1$ \\
\hline $331.4+00.5$ & He $2-145$ & $16.8 \times 15.5$ \\
\hline $331.5-02.7$ & He 2-161 & $16.3 \times 9.7$ \\
\hline $331.5-03.9$ & He 2-165 & $56.4 \times 46.3$ \\
\hline $331.7-01.0$ & Mz 3 & $47.8 \times 22.6$ \\
\hline $332.0-03.3$ & He $2-164$ & $16.4 \times 15.3$ \\
\hline $332.3-04.2$ & He $2-170$ & 1.3 \\
\hline $332.9-09.9$ & He 3-1333 & $3.2 \times 2.8$ \\
\hline $333.4+01.1$ & He $2-152$ & $11.8 \times 10.0$ \\
\hline $334.3-09.3$ & IC 4642 & $24.1 \times 21.7$ \\
\hline $334.8-07.4$ & SaSt 2-12 & $15.9 \times 11.9$ \\
\hline $335.4+09.2$ & ESO 330-02 & $30.8 \times 28.8$ \\
\hline $335.4-01.1$ & He 2-169 & $22.3 \times 18.5$ \\
\hline $336.2+01.9$ & Pe 1- 6 & $10.2 \times 8.7$ \\
\hline $336.2-06.9$ & PC 14 & $7.2 \times 5.1$ \\
\hline $336.3-05.6$ & He 2-186 & $2.9 \times \quad 1.4$ \\
\hline $336.8-07.2$ & K 2-17 & $39.3 \times 32.4$ \\
\hline $337.4+01.6$ & Pe 1- 7 & 2.4 \\
\hline $338.1-08.3$ & NGC 6326 & $20.6 \times 13.7$ \\
\hline $338.8+05.6$ & He $2-155$ & $16.9 \times 14.5$ \\
\hline $340.8+10.8$ & Lo 12 & $84.5 \times 70.4$ \\
\hline $340.8+12.3$ & Lo 11 & $65.7 \times 57.0$ \\
\hline $341.6+13.7$ & NGC 6026 & $53.0 \times 45.5$ \\
\hline $341.8+05.4$ & NGC 6153 & $28.0 \times 24.2$ \\
\hline $342.1+10.8$ & NGC 6072 & $74.3 \times 65.1$ \\
\hline $342.1+27.5$ & Me 2-1 & $8.9 \times 8.6$ \\
\hline $342.9-02.0$ & Pe $1-8$ & $24.3 \times 22.0$ \\
\hline $342.9-04.9$ & He 2-207 & $37.7 \times 26.0$ \\
\hline $343.4+11.9$ & H 1- 1 & $3.1 \times 2.7$ \\
\hline $343.6+03.7$ & SuWt 3 & $31.9 \times 16.3$ \\
\hline $345.2-01.2$ & H 1- 7 & $10.6 \times 8.7$ \\
\hline $345.2-08.8$ & Tc 1 & $12.9 \times 12.2$ \\
\hline $345.4+00.1$ & IC 4637 & $18.9 \times 13.5$ \\
\hline $346.2-08.2$ & IC 4663 & $20.1 \times 14.6$ \\
\hline $348.0-13.8$ & IC 4699 & $12.6 \times 8.0$ \\
\hline 349.3-01.1 & NGC 6337 & $47.6 \times 46.5$ \\
\hline $349.5+01.0$ & NGC 6302 & $89.9 \times 34.8$ \\
\hline $350.9+04.4$ & H 2- 1 & $4.3 \times \quad 3.7$ \\
\hline $352.9+11.4$ & K 2-16 & $26.6 \times 24.3$ \\
\hline $355.1-06.9$ & M 3-21 & 2.8 \\
\hline $355.4-04.0$ & Hf $2-1$ & $17.7 \times 14.6$ \\
\hline $356.5-02.3$ & M 1-27 & $6.7 \times 6.4$ \\
\hline $356.7-06.4$ & H 1-51 & $17.7 \times 15.2$ \\
\hline $357.0+02.4$ & M 4- 4 & $6.3 \times \quad 5.1$ \\
\hline $357.1-04.7$ & H 1-43 & 2.0 \\
\hline $357.3+04.0$ & H 2- 7 & $5.7 \times \quad 4.4$ \\
\hline
\end{tabular}


Table 2. continued.

\begin{tabular}{llr}
\hline \hline \multicolumn{1}{c}{$(1)$} & \multicolumn{1}{c}{$(2)$} & \multicolumn{1}{c}{$(3)$} \\
\hline $357.6-03.3$ & H 2-29 & $10.7 \times 9.8$ \\
$357.9-03.8$ & H 2-30 & $13.3 \times 13.3$ \\
$358.3-21.6$ & IC 1297 & $10.8 \times 9.8$ \\
$358.5+05.4$ & M 3-39 & $25.9 \times 18.1$ \\
$358.5-07.3$ & NGC 6563 & $59.1 \times 43.1$ \\
$358.6+01.8$ & M 4- 6 & $2.5 \times 2.3$ \\
$358.6-05.5$ & M 3-51 & $20.9 \times 14.5$ \\
$358.9-00.7$ & M 1-26 & $6.4 \times 6.0$ \\
$359.0-04.1$ & M 3-48 & $5.4 \times 4.4$ \\
$359.1+15.1$ & A 40 & $34.3 \times 30.4$ \\
$359.2+01.2$ & 19W32 & $23.6 \times 5.5$ \\
$359.3-00.9$ & Hb 5 & $51.7 \times 18.1$ \\
$359.4-03.4$ & H 2-33 & $7.8 \times 7.4$ \\
$359.9+05.1$ & M 3-9 & $17.2 \times 15.1$ \\
\hline
\end{tabular}

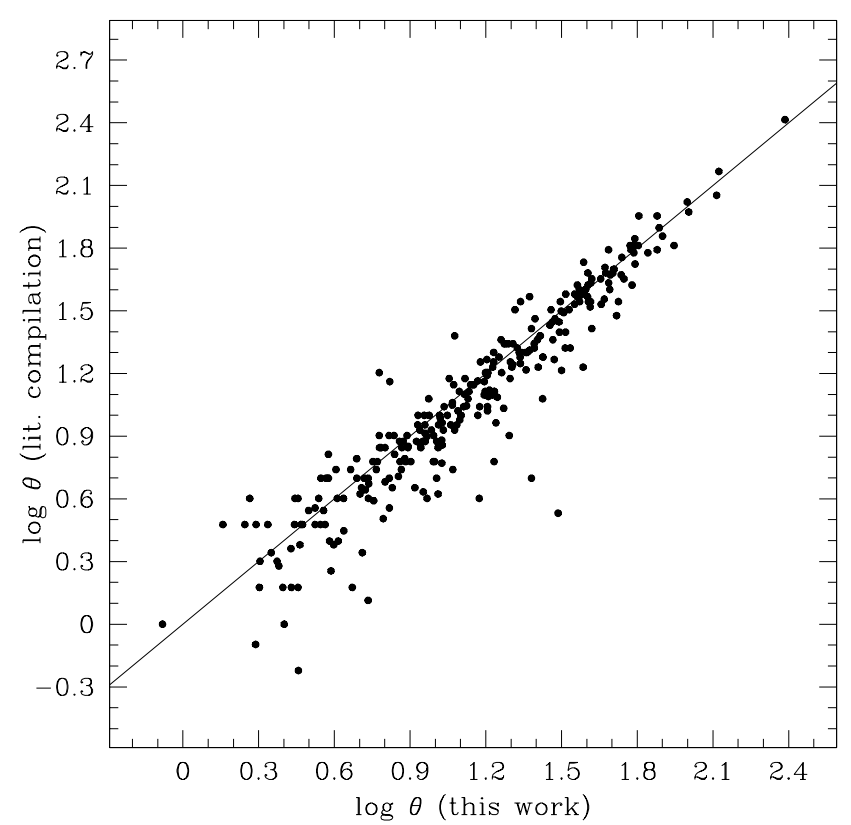

Fig. 9. Comparison of the PN diameters from the literature compilation (see text) with the diameters from this work (geometric means from the two dimensions given in Table 2). Solid line: 1 to 1 relation.

the case of larger nebulae usually concern bipolar nebulae having a compact core, e.g. IC $4634(000.3+12.2)$, M328 (021.8-00.4), M 2-48 (062.4-00.2), He 2-104 (315.4+09.4), $\mathrm{Hb} 5$ (359.3-00.9). The published diameters from VLA or optical measurements apparently referred to the core whereas the $10 \%$ contour from our images encompassed a significant portion of the outer bipolar structures.

In Fig. 10 we have compared the diameters from Table 2 with the results of VLA surveys of Aaquist \& Kwok (1990) and Zijlstra et al. (1989). Aaquist \& Kwok observed mainly compact PNe. They have applied three methods for deriving the PN diameters but it is not clear which method(s) has been used to measure a particular object. As can be seen from Fig. 10, our diameters are systematically larger than those of Aaquist \& Kwok, on average by factor 1.7. The agreement of our results with those of Zijlstra et al. is better especially if

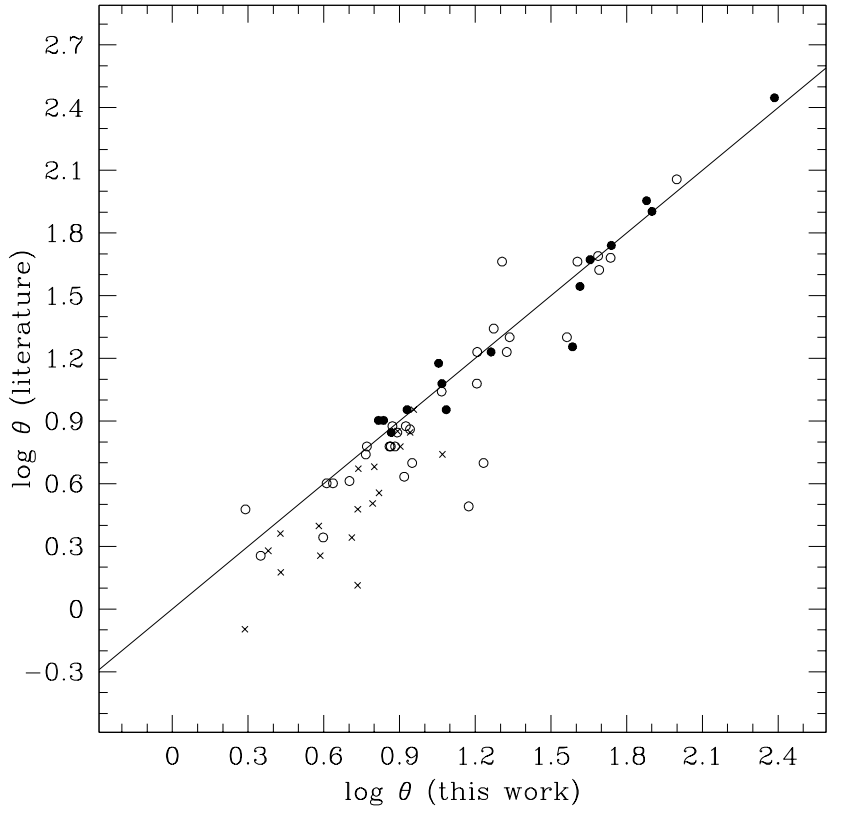

Fig. 10. Comparison of the PN diameters from Aaquist \& Kwok (1990) (crosses) and Zijlstra et al. (1989) (full circles - from 10\% contour, open circles - from Gaussian deconvolution) with the diameters from this work (geometric means from the two dimensions given in Table 2). Solid line: 1 to 1 relation.

nebulae greater than $\sim 10$ arcsec are considered and the diameter of Zijlstra et al. resulted from their $10 \%$ contour (full circles in the figure). In four cases the discrepancy is larger than factor 2. All the cases concern bipolar nebulae. For three of them, i.e already mentioned M3-28, M2-48, as well as NGC 2440 $(234.8+02.4)$, our diameters are larger and the reason has been explained above. In the case of M2-9 our diameter is significantly smaller and it seems that Zijlstra et al. measured the angular extend of the elongated, bipolar lobes whereas our value is a geometric mean from the major and minor axis measurements.

\section{Discussion and conclusions}

We have measured angular dimensions for $312 \mathrm{PNe}$. As a primary definition of the PN size we have adopted the extension of nebular emission up to the $10 \%$ level of the maximum surface brightness. This seems to be a reasonable choice for extended, well resolved nebulae. For partly resolved objects deconvolution methods have to be used. So far the Gaussian deconvolution has primarily been applied.

We have, however, found that for compact nebulae the $10 \%$ contour measurements can also give satisfactory results provided that they are corrected for the finite resolution using Eq. (2). As has been shown in Sects. 5.2 and 5.4 this simple deconvolution of the $10 \%$ contour measurments makes the resulting diameters reliable down to nebulae as compact as $\log \Theta_{10 \%, \mathrm{~d}} / \Phi_{\mathrm{b}} \simeq 0.3$ (or equivalently $\log \Phi_{\mathrm{d}}($ Gauss $\left.) / \Phi_{\mathrm{b}} \simeq 0.05\right)$. The derivation of $\Theta_{10 \%, \mathrm{~d}}$ has several advantages over the deconvolution methods. It is simpler to apply. No assumption has to be made about the surface brightness distribution. It is a more universal method as it can 
be applied to compact and partially resolved PNe as well as to extended and well resolved nebulae where the deconvolution methods usually fail in practice.

The Gaussian deconvolution gives fairly reliable and stable results for partly resolved nebulae. However it systematically underestimates the diameters compared to the $10 \%$ contour measurements. The difference depends on the model nebula adopted in the Gaussian deconvolution. It is only 5-7\% for the constant emissivity sphere but increases up to $45 \%$ for a thin shell. This result means that in catalogues and data bases the dimensions of compact PNe, usually derived from the Gaussian deconvolution, are probably underestimated compared to those of extended nebulae. It is difficult to estimate this systematic difference. The deconvolution procedures used in observational surveys, like in Aaquist \& Kwok (1990) or Zijlstra et al. (1989), were significantly simplified compared to that of van Hoof (2000). Usually the same value of the conversion factor has been used for all the objects. This certainly introduced additional uncertainties in the final results.

The second-moment deconvolution appeared to give results often uncertain and in poor agreement with the results from the other two methods, in particular for compact nebulae.

Our study does not solve definitevely the problem of measuring the PN dimensions. It makes however an important step in the field in the sense that it gives a quantity which means physically the same and is comparable for all the objects in our sample. One has, however, to remember that the (faint) nebular emission can extend well beyond the diameters given in Table 2. This can be seen for many objects in our sample by comparing Cols. (9) and (8) in Table 1.

Acknowledgements. We are very grateful to the referee (J.J. Condon) whose comments resulted in a significant improvement of the work reported in this paper. This work has partly been supported from a grant No. 2.P03D.020.17 financed by the Polish State Committee for Scientific Research.

\section{References}

Aaquist, O. B., \& Kwok, S. 1990, A\&AS, 84, 229

Acker, A., Ochsenbein, F., Stenholm, B., et al. 1992, Strasbourg-ESO Catalogue of Galactic Planetary Nebulae (ESO publication)

Górny, S. K., Schwarz, H. E., Corradi, R. L. M., \& van Winckel, H. 1999, A\&AS, 136, 145

Manchado, A., Guerrero, M. A., Stanghellini, L., \& Serra-Ricart, M. 1996, The IAC Morphological Catalog of Northern Galactic Planetary Nebulae, Instituto de Astrofisica de Canarias

Schwarz, H. E., Corradi, R. L. M., \& Melnick, J. 1992, A\&AS, 96, 23

Siódmiak, N., \& Tylenda, R. 2001, A\&AS, 373, 1032

van Hoof, P. A. M. 2000, MNRAS, 314, 99

Zijlstra, A. A., Pottasch, S. R., \& Bignell, C. 1989, A\&AS, 79, 329 
R. Tylenda et al.: Angular dimensions of planetary nebulae, Online Material p 1

\section{Online Material}


R. Tylenda et al.: Angular dimensions of planetary nebulae, Online Material p 2

Table 1. Results of measurements (see text for explanation of the columns).

\begin{tabular}{|c|c|c|c|c|c|c|c|c|c|c|c|c|}
\hline \multirow{2}{*}{$\begin{array}{c}\text { PN G } \\
1 \\
000.1+17.2\end{array}$} & \multirow{2}{*}{$\begin{array}{c}\text { Main Name } \\
2 \\
\text { PC } 12\end{array}$} & \multirow{2}{*}{$\begin{array}{c}\text { figure } \\
3 \\
\text { s } 40\end{array}$} & \multirow{2}{*}{$\begin{array}{c}\Phi_{d, G} \\
4 \\
1.4 \times 1.3\end{array}$} & \multirow{2}{*}{$\begin{array}{c}\Phi_{b, G} \\
5 \\
0.8 \times 0.7\end{array}$} & \multicolumn{2}{|c|}{$\begin{array}{c}\Phi_{d, s m} \\
6\end{array}$} & \multirow{2}{*}{$\begin{array}{c}\Phi_{b, s m} \\
7 \\
0.8 \times 0.8\end{array}$} & \multicolumn{2}{|c|}{$\begin{array}{c}10 \% \text { level } \\
8\end{array}$} & \multicolumn{2}{|c|}{$\begin{array}{c}3 \sigma \text { level } \\
9\end{array}$} & $\begin{array}{c}\% 3 \sigma \\
10 \\
\end{array}$ \\
\hline & & & & & $2.0 \mathrm{x}$ & 1.5 & & $2.7 x$ & 2.6 & & & \\
\hline 000.1-01.1 & M 3-43 & s 56 & $2.2 \times 1.5$ & $1.4 \times 1.5$ & $2.2 \mathrm{x}$ & 1.5 & $1.3 \times 1.6$ & $4.8 \mathrm{x}$ & 3.7 & & & \\
\hline 000.1-05.6 & H 2-40 & s 61 & & $1.7 \times 1.5$ & $8.9 \mathrm{x}$ & 8.5 & $1.8 \times 1.6$ & $18.5 \mathrm{x}$ & 17.2 & & & \\
\hline 000.2-01.9 & M 2-19 & g 28 & $5.4 \times 4.5$ & $1.9 \times 1.7$ & $5.1 \mathrm{x}$ & 6.0 & $1.8 \times 1.7$ & $10.0 \mathrm{x}$ & 9.0 & $19.0 \mathrm{x}$ & 12.7 & 1.5 \\
\hline $000.3+12.2$ & IC 4634 & s 42 & & $0.8 \times 0.8$ & & & $0.8 \times 0.8$ & $20.6 x$ & 6.8 & $24.0 \mathrm{x}$ & 19.0 & 0.3 \\
\hline 000.4-01.9 & M 2-20 & s 56 & $2.0 \times 1.9$ & $1.4 \times 1.2$ & $2.8 \mathrm{x}$ & 2.5 & $1.3 \times 1.1$ & $4.7 \mathrm{x}$ & 4.2 & & & \\
\hline $000.7+04.7$ & H 2-11 & s 49 & $1.3 \times 1.1$ & $1.0 \times 1.2$ & $1.5 \mathrm{x}$ & 1.3 & $1.0 \times 1.2$ & $2.9 \mathrm{x}$ & 2.6 & & & \\
\hline $001.0+01.9$ & K 1- 4 & s 52 & & $1.9 \times 1.4$ & & & $1.4 \times 1.3$ & $48.2 \mathrm{x}$ & 33.8 & & & \\
\hline $001.5-06.7$ & SwSt 1 & s 66 & $3.3 \times 3.1$ & $1.1 \times 1.3$ & $3.9 \mathrm{x}$ & 4.1 & $1.3 \times 1.4$ & $6.0 \mathrm{x}$ & 5.7 & & & \\
\hline $002.0-13.4$ & IC 4776 & s 74 & $4.1 \times 3.2$ & $1.2 \times 1.3$ & $4.5 \mathrm{x}$ & 3.1 & $1.3 \times 1.4$ & $8.8 \mathrm{x}$ & 4.7 & $22.1 \mathrm{x}$ & 18.2 & 0.1 \\
\hline $002.2-06.3$ & H 1-63 & s 66 & $2.1 \times 1.7$ & $1.7 \times 1.5$ & $2.5 \mathrm{x}$ & 2.2 & $1.7 \times 1.6$ & $4.9 \mathrm{x}$ & 4.2 & & & \\
\hline $002.2-09.4$ & Cn 1-5 & s 69 & $4.6 \times 4.3$ & $0.7 \times 0.8$ & $4.2 \mathrm{x}$ & 4.0 & $0.9 \times 0.9$ & $7.5 \mathrm{x}$ & 7.5 & $29.9 \mathrm{x}$ & 27.6 & 0.1 \\
\hline $002.4+05.8$ & NGC 6369 & s 49 & & $1.5 \times 1.2$ & & & $1.2 \times 1.0$ & $33.1 \mathrm{x}$ & 32.8 & $64.7 x$ & 41.4 & 0.9 \\
\hline $002.5-01.7$ & Pe $2-11$ & s 58 & & $1.5 \times 1.4$ & $3.9 x$ & 3.4 & $1.6 \times 1.5$ & $8.3 \mathrm{x}$ & 7.0 & & & \\
\hline $002.7-04.8$ & M 1-42 & s 63 & & $1.4 \times 1.6$ & $7.2 \mathrm{x}$ & 6.8 & $1.4 \times 1.6$ & $13.3 \mathrm{x}$ & 11.7 & & & \\
\hline $002.7-52.4$ & IC $5148-50$ & g 9 & & & & & & $132.5 \mathrm{x}$ & 127.8 & & & \\
\hline $002.8+01.7$ & H 2-20 & s 53 & st. & $2.1 \times 1.8$ & $1.6 \mathrm{x}$ & 1.4 & $1.7 \times 1.5$ & $3.9 \mathrm{x}$ & 3.1 & & & \\
\hline $003.1+02.9$ & $\mathrm{Hb} 4$ & s 52 & $5.8 \times 4.7$ & $1.5 \times 1.6$ & $5.9 \mathrm{x}$ & 4.1 & $1.4 \times 1.4$ & $11.4 \mathrm{x}$ & 7.4 & & & \\
\hline $003.1+03.4$ & H 2-17 & s 51 & $2.6 \times 2.3$ & $1.5 \times 1.4$ & $2.4 \mathrm{x}$ & 2.2 & $1.7 \times 1.4$ & $5.5 \mathrm{x}$ & 4.7 & & & \\
\hline $003.5-02.4$ & IC 4673 & s 60 & & $0.9 \times 0.9$ & $10.7 \mathrm{x}$ & 8.3 & $1.1 \times 1.1$ & $22.1 \mathrm{x}$ & 15.3 & & & \\
\hline 003.5-04.6 & NGC 6565 & s 63 & & $1.7 \times 1.6$ & $6.5 \mathrm{x}$ & 6.2 & $1.7 \times 1.5$ & $11.2 \mathrm{x}$ & 10.9 & & & \\
\hline 003.6-02.3 & M 2-26 & s 59 & $10.2 \times 6.1$ & $1.1 \times 1.1$ & $6.0 \mathrm{x}$ & 5.3 & $1.1 \times 1.1$ & $10.7 \mathrm{x}$ & 10.6 & & & \\
\hline $003.7+07.9$ & H 2- 8 & s 47 & $3.6 \times 3.0$ & $0.8 \times 0.9$ & $8.2 \mathrm{x}$ & 3.5 & $0.9 \times 0.9$ & $12.0 \mathrm{x}$ & 6.9 & & & \\
\hline 003.8-04.5 & H 2-41 & s 64 & & $1.4 \times 1.5$ & $5.1 \mathrm{x}$ & 4.6 & $1.4 \times 1.5$ & $9.7 \mathrm{x}$ & 9.6 & & & \\
\hline 003.8-17.1 & $\mathrm{Hb} 8$ & s 76 & $0.6 \times 0.4$ & $1.4 \times 1.4$ & $1.5 \mathrm{x}$ & 1.3 & $1.3 \times 1.3$ & $2.9 \mathrm{x}$ & 2.3 & & & \\
\hline \multirow[t]{2}{*}{ 003.9-14.9 } & $\mathrm{Hb} 7$ & g 13 & $1.7 \times 1.7$ & $1.7 \times 1.9$ & $3.0 \mathrm{x}$ & 2.8 & $1.7 \times 1.8$ & $4.3 \mathrm{x}$ & 4.3 & & & \\
\hline & & s 74 & $2.2 \times 1.8$ & $1.3 \times 1.2$ & $4.1 \mathrm{x}$ & 2.4 & $1.4 \times 1.3$ & $5.5 \mathrm{x}$ & 4.2 & & & \\
\hline 004.0-11.1 & M 3-29 & s 72 & $5.6 \times 5.4$ & $1.3 \times 1.2$ & $4.7 \mathrm{x}$ & 5.2 & $1.4 \times 1.3$ & $9.9 \mathrm{x}$ & 8.9 & $15.1 \mathrm{x}$ & 12.2 & 1.4 \\
\hline 004.8-22.7 & He $2-436$ & g 24 & $1.8 \times 1.7$ & $1.5 \times 1.0$ & $2.1 \mathrm{x}$ & 2.0 & $1.5 \times 1.2$ & $4.0 \mathrm{x}$ & 4.0 & & & \\
\hline 004.9-04.9 & M 1-44 & s 66 & $4.0 \times 3.6$ & $1.3 \times 1.5$ & $5.2 \mathrm{x}$ & 4.4 & $1.3 \times 1.5$ & $6.5 \mathrm{x}$ & 6.0 & $19.9 \mathrm{x}$ & 12.2 & 0.5 \\
\hline $005.0+03.0$ & Pe 1- 9 & s 53 & & $1.9 \times 1.8$ & $6.8 \mathrm{x}$ & 6.7 & $1.8 \times 1.7$ & $14.0 \mathrm{x}$ & 13.8 & & & \\
\hline 005.0-03.9 & H 2-42 & s 64 & & $1.4 \times 1.5$ & $6.3 \mathrm{x}$ & 6.2 & $1.4 \times 1.6$ & $13.3 \mathrm{x}$ & 12.2 & & & \\
\hline 005.1-08.9 & Hf $2-2$ & s 69 & & $1.0 \times 1.0$ & $11.6 \mathrm{x}$ & 11.3 & $1.1 \times 1.1$ & $21.8 \mathrm{x}$ & 21.8 & & & \\
\hline $006.0+03.1$ & M 1-28 & g 1 & & $1.6 \times 1.6$ & & & $1.9 \times 1.7$ & $33.2 \mathrm{x}$ & 30.4 & $64.8 \mathrm{x}$ & 41.0 & 4.2 \\
\hline $006.1+08.3$ & M 1-20 & s 49 & $1.3 \times 1.3$ & $0.8 \times 1.0$ & $1.5 \mathrm{x}$ & 1.4 & $0.9 \times 1.0$ & $2.9 \mathrm{x}$ & 2.9 & & & \\
\hline 006.8-08.6 & $\mathrm{Al} 1$ & s 71 & & $1.7 \times 1.5$ & $7.7 \mathrm{x}$ & 6.4 & $1.8 \times 1.6$ & $15.2 \mathrm{x}$ & 12.7 & & & \\
\hline $007.2+01.8$ & $\mathrm{Hb} 6$ & s 57 & $4.5 \times 4.4$ & $1.2 \times 1.4$ & $4.1 \mathrm{x}$ & 3.8 & $1.3 \times 1.6$ & $8.0 \mathrm{x}$ & 7.3 & & & \\
\hline $008.0+03.9$ & NGC 6445 & s 55 & & $1.1 \times 1.0$ & & & $1.2 \times 1.1$ & $45.3 \mathrm{x}$ & 36.2 & $188.0 \mathrm{x}$ & 116.7 & 0.5 \\
\hline 008.3-01.1 & M 1-40 & s 62 & $4.8 \times 3.8$ & $1.8 \times 1.6$ & $5.3 \mathrm{x}$ & 6.0 & $1.7 \times 1.6$ & $9.7 \mathrm{x}$ & 8.1 & $20.8 \mathrm{x}$ & 16.4 & 0.5 \\
\hline 008.3-07.3 & NGC 6644 & s 70 & $2.4 \times 2.3$ & $1.6 \times 1.5$ & $2.6 \mathrm{x}$ & 2.4 & $1.8 \times 1.6$ & $5.2 \mathrm{x}$ & 5.2 & & & \\
\hline 009.4-05.0 & NGC 6629 & s 68 & & $0.7 \times 0.9$ & $8.8 \mathrm{x}$ & 8.3 & $0.8 \times 0.9$ & $16.6 \mathrm{x}$ & 15.6 & $40.0 \mathrm{x}$ & 38.2 & 0.5 \\
\hline 009.4-09.8 & M 3-32 & g 29 & $4.5 \times 3.4$ & $2.2 \times 2.3$ & $4.2 \mathrm{x}$ & 3.5 & $2.1 \times 2.0$ & $9.0 \mathrm{x}$ & 8.0 & & & \\
\hline $009.6+10.5$ & A 41 & s 50 & & $1.5 \times 1.3$ & $10.5 \mathrm{x}$ & 10.4 & $1.6 \times 1.5$ & $20.3 x$ & 17.5 & & & \\
\hline $009.6+14.8$ & NGC 6309 & s 46 & & $0.9 \times 1.2$ & $12.5 \mathrm{x}$ & 8.4 & $0.9 \times 1.2$ & $22.9 \mathrm{x}$ & 12.6 & $52.3 \mathrm{x}$ & 26.5 & 0.7 \\
\hline 009.6-10.6 & M 3-33 & g 29 & $4.4 \times 3.8$ & $1.7 \times 1.6$ & $4.3 \mathrm{x}$ & 3.9 & $1.5 \times 1.4$ & $8.0 \mathrm{x}$ & 7.9 & & & \\
\hline 010.7-06.4 & IC 4732 & s 71 & $1.0 \times 0.9$ & $1.8 \times 1.9$ & $1.8 \mathrm{x}$ & 1.7 & $1.7 \times 1.9$ & $3.6 \mathrm{x}$ & 3.4 & & & \\
\hline $010.8+18.0$ & M 2- 9 & s 44 & & $1.2 \times 0.9$ & & & $1.2 \times 0.9$ & $37.0 \mathrm{x}$ & 11.2 & $56.0 \mathrm{x}$ & 14.8 & 0.7 \\
\hline 010.8-01.8 & NGC 6578 & s 67 & $7.1 \times 6.8$ & $1.8 \times 1.7$ & $6.3 x$ & 6.2 & $1.9 \times 1.7$ & $12.5 \mathrm{x}$ & 12.2 & & & \\
\hline $011.0-05.1$ & M 1-47 & g 27 & $3.3 \times 2.9$ & $1.5 \times 2.1$ & $3.8 \mathrm{x}$ & 3.3 & $1.5 \times 1.9$ & $6.8 \mathrm{x}$ & 6.5 & & & \\
\hline $011.1+11.5$ & M 2-13 & s 48 & $1.5 \times 1.4$ & $0.9 \times 0.9$ & $2.3 \mathrm{x}$ & 1.7 & $1.0 \times 1.0$ & $3.4 \mathrm{x}$ & 2.9 & & & \\
\hline 011.7-00.6 & NGC 6567 & s 65 & $4.8 \times 3.9$ & $1.6 \times 1.5$ & $4.7 \mathrm{x}$ & 3.6 & $1.8 \times 1.6$ & $8.6 \mathrm{x}$ & 7.0 & & & \\
\hline $011.9+04.2$ & M 1-32 & s 57 & $5.1 \times 2.2$ & $1.4 \times 1.2$ & $5.1 \mathrm{x}$ & 5.0 & $1.4 \times 1.2$ & $9.4 \mathrm{x}$ & 8.3 & $18.3 \mathrm{x}$ & 17.4 & 0.2 \\
\hline $012.5-09.8$ & M 1-62 & g 27 & $2.6 \times 2.5$ & $1.9 \times 1.8$ & $2.9 \mathrm{x}$ & 2.8 & $1.9 \times 1.8$ & $5.8 \mathrm{x}$ & 5.8 & & & \\
\hline 013.0-04.3 & Pe 2-14 & g 34 & $2.8 \times 2.7$ & $2.1 \times 1.5$ & $2.6 \mathrm{x}$ & 2.9 & $1.8 \times 1.5$ & $6.5 \mathrm{x}$ & 6.1 & & & \\
\hline $013.3+32.7$ & Sn 1 & s 38 & $3.0 \times 2.8$ & $1.6 \times 1.5$ & $3.5 \mathrm{x}$ & 3.0 & $1.4 \times 1.4$ & $6.6 \mathrm{x}$ & 5.7 & & & \\
\hline 014.6-04.3 & M 1-50 & s 70 & $2.1 \times 2.1$ & $1.7 \times 1.6$ & $2.4 \mathrm{x}$ & 2.3 & $1.7 \times 1.6$ & $5.2 \mathrm{x}$ & 4.9 & & & \\
\hline 016.4-01.9 & M 1-46 & s 68 & & $1.7 \times 1.7$ & $6.7 \mathrm{x}$ & 6.2 & $1.5 \times 1.4$ & $12.5 \mathrm{x}$ & 11.7 & & & \\
\hline
\end{tabular}


Table 1. continued.

\begin{tabular}{|c|c|c|c|c|c|c|c|c|c|c|}
\hline$\overline{1.1}$ & $\overline{2}$ & 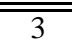 & 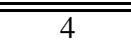 & $\overline{5}$ & $\overline{\overline{6}}$ & $\overline{77}$ & $\overline{8}$ & & $\overline{99}$ & $\overline{10}$ \\
\hline $017.6-10.2$ & A 51 & s 75 & & $1.4 \times 1.3$ & & $1.4 \times 1.4$ & $59.3 x$ & 59.0 & & \\
\hline 017.9-04.8 & M 3-30 & s 73 & & $1.0 \times 1.0$ & & $1.1 \times 1.1$ & $19.2 \mathrm{x}$ & 18.5 & & \\
\hline 020.9-01.1 & M 1-51 & g 9 & $7.2 \times 6.2$ & $1.8 \times 2.1$ & $8.4 \times \quad 5.1$ & $2.1 \times 2.5$ & $15.9 \times$ & 8.9 & & \\
\hline $021.2-03.9$ & We 1-7 & s 73 & & $1.1 \times 1.1$ & & $1.2 \times 1.1$ & $20.6 x$ & 19.8 & & \\
\hline $021.7-00.6$ & M 3-55 & s 70 & $6.4 \times 5.8$ & $1.7 \times 1.8$ & $6.3 \times 5.2$ & $1.7 \times 1.7$ & $12.6 \mathrm{x}$ & 9.8 & & \\
\hline \multirow[t]{2}{*}{$021.8-00.4$} & M 3-28 & & $9.0 \times 6.7$ & $1.9 \times 1.8$ & $10.7 \times 6.5$ & $2.0 \times 2.0$ & $20.9 \mathrm{x}$ & 12.3 & $33.2 \times 15.5$ & 2.8 \\
\hline & & & $8.1 \times 6.3$ & $1.0 \times 1.2$ & $8.2 \times 5.2$ & $1.2 \times 1.3$ & $27.6 \mathrm{x}$ & 12.6 & & \\
\hline $023.9-02.3$ & M 1-59 & & $3.6 \times 3.5$ & $1.5 \times 1.8$ & $5.5 \times 3.9$ & $1.6 \times 1.8$ & $7.2 \mathrm{x}$ & 6.8 & $24.1 \times 16.5$ & 0.2 \\
\hline \multirow[t]{2}{*}{$024.2+05.9$} & M 4- 9 & & & $1.5 \times 1.1$ & & $1.5 \times 1.2$ & $47.9 \mathrm{x}$ & 42.0 & & \\
\hline & & s 65 & & $1.8 \times 1.7$ & & $1.8 \times 1.7$ & $48.1 \mathrm{x}$ & 43.4 & & \\
\hline $024.3-03.3$ & Pe 1-17 & & $6.0 \times 3.6$ & $1.2 \times 1.2$ & $7.8 \times \quad 4.0$ & $1.5 \times 1.5$ & $14.9 \mathrm{x}$ & 7.9 & & \\
\hline $025.8-17.9$ & NGC 6818 & s 84 & & $0.9 \times 0.8$ & $13.9 \times 12.9$ & $1.0 \times 1.0$ & $24.7 \mathrm{x}$ & 24.7 & $35.1 \times 32.2$ & 0.4 \\
\hline 025.9-10.9 & $\mathrm{Na} 2$ & s 80 & $3.9 \times 3.3$ & $0.8 \times 0.9$ & $3.2 \times 3.7$ & $0.9 \times 1.1$ & $6.5 \mathrm{x}$ & 5.9 & & \\
\hline 027.3-03.4 & A 49 & s 74 & & $1.0 \times 0.8$ & & $0.9 \times 0.9$ & $54.1 \mathrm{x}$ & 38.2 & & \\
\hline $027.7+00.7$ & M 2-45 & s 72 & $5.4 \times 5.2$ & $1.2 \times 1.1$ & $4.5 \times 3.9$ & $1.2 \times 1.2$ & $9.6 \mathrm{x}$ & 7.8 & & \\
\hline $028.0+10.2$ & WeSb 3 & s 61 & & $1.7 \times 1.7$ & & $1.5 \times 1.4$ & $50.8 \mathrm{x}$ & 43.2 & & \\
\hline 028.7-03.9 & Pe 1-21 & & & $1.7 \times 1.1$ & $5.6 \times \quad 4.9$ & $1.6 \times 1.2$ & $11.5 \mathrm{x}$ & 10.6 & & \\
\hline $029.2-05.9$ & NGC 6751 & s 76 & & $1.1 \times 1.1$ & $15.9 \times 14.1$ & $1.3 \times 1.3$ & $24.2 \mathrm{x}$ & 23.3 & $55.4 \times 54.1$ & 0.6 \\
\hline $030.8+03.4$ & A 47 & s 72 & & $1.5 \times 1.3$ & & $1.3 \times 1.1$ & $17.7 \mathrm{x}$ & 12.6 & & \\
\hline $031.0-10.8$ & M 3-34 & s 82 & $4.3 \times 3.8$ & $0.9 \times 1.0$ & $4.0 \times 3.3$ & $1.0 \times 1.1$ & $7.6 \mathrm{x}$ & 6.6 & $10.7 \times 9.3$ & 0.6 \\
\hline $033.0-05.3$ & A 55 & s 77 & & $1.7 \times 1.6$ & & $1.7 \times 1.6$ & $56.9 \mathrm{x}$ & 52.4 & & \\
\hline $033.1-06.3$ & NGC 6772 & s 79 & & $1.8 \times 1.6$ & & $1.6 \times 1.5$ & $80.8 \mathrm{x}$ & 70.9 & & \\
\hline $033.8-02.6$ & NGC 6741 & s 75 & $5.3 \times 4.4$ & $1.3 \times 1.4$ & $5.2 \times 3.8$ & $1.3 \times 1.3$ & $9.4 \mathrm{x}$ & 7.0 & & \\
\hline $034.5-06.7$ & NGC 6778 & s 80 & & $1.1 \times 0.9$ & $13.2 \times 10.9$ & $1.0 \times 0.9$ & $21.5 \mathrm{x}$ & 15.6 & $39.5 \times 31.0$ & 0.6 \\
\hline 035.9-01.1 & Sh 2- 71 & & & $1.3 \times 1.1$ & & $1.5 \times 1.4$ & $132.4 \mathrm{x}$ & 74.9 & 168.4 x 85.1 & 5.1 \\
\hline $037.5-05.1$ & A 58 & s 80 & & $1.1 \times 1.0$ & & $1.1 \times 1.0$ & $44.7 \mathrm{x}$ & 33.7 & & \\
\hline $037.7-34.5$ & NGC 7009 & s 85 & & $1.4 \times 1.0$ & $14.3 \times 11.4$ & $1.0 \times 0.7$ & $28.1 \mathrm{x}$ & 22.1 & $55.0 \times 29.6$ & 0.6 \\
\hline $037.8-06.3$ & NGC 6790 & s 81 & $2.5 \times 2.1$ & $0.9 \times 0.7$ & $2.7 \times \quad 2.5$ & $0.9 \times 0.7$ & $4.7 \mathrm{x}$ & 3.6 & & \\
\hline $038.1-25.4$ & A 70 & s 85 & & $1.2 \times 1.2$ & & $1.4 \times 1.3$ & $45.2 \mathrm{x}$ & 37.9 & & \\
\hline $038.2+12.0$ & Cn 3-1 & s 67 & $3.3 \times 3.2$ & $0.9 \times 1.1$ & $3.1 \times 3.2$ & $0.9 \times 0.9$ & $6.0 \mathrm{x}$ & 4.9 & & \\
\hline $039.5-02.7$ & M 2-47 & s 78 & $4.0 \times 2.8$ & $1.6 \times 1.9$ & $3.8 \times 3.1$ & $1.5 \times 1.8$ & $7.5 \mathrm{x}$ & 6.0 & & \\
\hline $040.3-00.4$ & A 53 & s 76 & & $1.1 \times 1.2$ & $19.3 \times 18.8$ & $1.1 \times 1.1$ & $32.0 \mathrm{x}$ & 31.2 & & \\
\hline 042.9-06.9 & NGC 6807 & s 83 & $1.3 \times 1.3$ & $1.0 \times 0.9$ & $1.6 \times 1.6$ & $1.1 \times 1.1$ & $2.6 \mathrm{x}$ & 2.6 & & \\
\hline $043.1+03.8$ & M 1-65 & s 75 & $2.3 \times 2.3$ & $1.2 \times 1.4$ & $2.6 \times 2.5$ & $1.1 \times 1.3$ & $4.7 x$ & 4.7 & $12.0 \times 12.2$ & 0.3 \\
\hline $043.3+11.6$ & M 3-27 & s 69 & $1.4 \times 1.2$ & $1.1 \times 1.2$ & $1.8 \times \quad 1.8$ & $1.3 \times 1.3$ & $3.5 \mathrm{x}$ & 3.1 & & \\
\hline $045.4-02.7$ & Vy $2-2$ & s 81 & $1.6 \times 1.5$ & $0.9 \times 1.0$ & $2.1 \times 1.9$ & $0.8 \times 0.9$ & $3.6 \mathrm{x}$ & 3.1 & $13.0 \times 12.5$ & 0.1 \\
\hline $045.7-04.5$ & NGC 6804 & s 83 & & $1.3 \times 1.1$ & & $1.4 \times 1.2$ & $58.3 \mathrm{x}$ & 48.6 & & \\
\hline 046.4-04.1 & NGC 6803 & s 83 & $3.2 \times 2.7$ & $1.0 \times 1.2$ & $3.2 \times 2.9$ & $1.1 \times 1.3$ & $5.7 \mathrm{x}$ & 5.5 & & \\
\hline $054.1-12.1$ & NGC 6891 & & $7.6 \times 6.4$ & $2.0 \times 1.6$ & $7.4 \times \quad 6.8$ & $2.2 \times 1.9$ & $14.0 \mathrm{x}$ & 13.0 & & \\
\hline 061.4-09.5 & NGC 6905 & & & $2.6 \times 2.3$ & & $2.6 \times 2.3$ & $43.5 \mathrm{x}$ & 35.9 & & \\
\hline $062.4-00.2$ & M 2-48 & & $9.3 \times 5.7$ & $2.6 \times 2.8$ & $10.5 \times 6.4$ & $2.3 \times 2.4$ & $20.0 x$ & 12.4 & $29.0 \times 15.6$ & 4.5 \\
\hline $065.2-05.6$ & He $1-6$ & & & $2.3 \times 2.2$ & & $2.5 \times 2.5$ & $40.7 \mathrm{x}$ & 21.9 & & \\
\hline $066.7-28.2$ & NGC 7094 & & & $1.5 \times 1.3$ & & $1.6 \times 1.5$ & $102.5 \mathrm{x}$ & 99.4 & & \\
\hline $069.2+03.8$ & K 3-46 & & & $2.2 \times 2.3$ & $21.4 \times \quad 9.7$ & $2.4 \times 2.5$ & $36.4 \mathrm{x}$ & 23.9 & & \\
\hline $069.4-02.6$ & NGC 6894 & & & $2.4 \times 2.3$ & & $2.4 \times 2.4$ & $56.6 \mathrm{x}$ & 53.5 & & \\
\hline $118.8-74.7$ & NGC 246 & g 30 & & & & & $260.4 x$ & 226.7 & & \\
\hline $161.2-14.8$ & IC 2003 & & $6.1 \times 4.8$ & $2.3 \times 2.6$ & $5.6 \times \quad 4.7$ & $2.2 \times 2.6$ & $10.8 \mathrm{x}$ & 9.4 & & \\
\hline $171.3-25.8$ & $\mathrm{Ba} 1$ & & & $2.1 \times 2.0$ & & $1.9 \times 1.9$ & $40.0 \mathrm{x}$ & 38.5 & & \\
\hline $174.2-14.6$ & Н 3-29 & & $9.7 \times 9.2$ & $2.5 \times 2.2$ & $7.8 \times \quad 5.6$ & $2.5 \times 2.3$ & $24.2 \mathrm{x}$ & 19.2 & & \\
\hline $189.1+19.8$ & NGC 2371-72 & & & & & & $48.9 \mathrm{x}$ & 30.6 & $130.9 \times 55.7$ & 1.9 \\
\hline $190.3-17.7$ & J 320 & s 1 & $5.8 \times 2.8$ & $2.0 \times 2.1$ & $5.3 \times 3.8$ & $1.9 \times 1.8$ & $10.1 \mathrm{x}$ & 7.4 & $22.4 \times 12.6$ & 0.7 \\
\hline $194.2+02.5$ & J 900 & s 5 & $4.6 \times 4.2$ & $2.0 \times 1.7$ & $4.5 \times \quad 4.7$ & $1.8 \times 1.6$ & $8.8 \mathrm{x}$ & 8.6 & & \\
\hline 196.6-10.9 & NGC 2022 & s 2 & & $1.5 \times 1.4$ & $14.5 \times 13.6$ & $1.3 \times 1.3$ & $28.0 \mathrm{x}$ & 25.6 & $32.0 \times 30.7$ & 2.6 \\
\hline $197.2-14.2$ & K 1- 7 & s 2 & & $1.4 \times 1.4$ & $19.6 \times 17.6$ & $1.3 \times 1.3$ & $37.3 \mathrm{x}$ & 36.1 & & \\
\hline $198.6-06.3$ & A 12 & s 3 & & $1.3 \times 1.2$ & $23.1 \times 22.8$ & $1.3 \times 1.2$ & $44.2 \mathrm{x}$ & 38.6 & & \\
\hline 201.9-04.6 & We 1-4 & s 4 & & $0.9 \times 0.9$ & & $1.0 \times 1.0$ & $41.4 \mathrm{x}$ & 37.6 & & \\
\hline $206.4-40.5$ & NGC 1535 & s 1 & & & & & $33.3 \mathrm{x}$ & 32.1 & $51.5 \times 49.1$ & 0.5 \\
\hline $212.0+04.3$ & M 1- 9 & & $1.5 \times 1.4$ & $2.2 \times 1.8$ & $2.1 \times 2.0$ & $2.1 \times 1.8$ & $4.7 \mathrm{x}$ & 4.3 & & \\
\hline $214.9+07.8$ & A 20 & s 8 & & $2.2 \times 2.0$ & & $2.2 \times 2.1$ & $67.4 \mathrm{x}$ & 60.6 & & \\
\hline
\end{tabular}


Table 1. continued.

\begin{tabular}{|c|c|c|c|c|c|c|c|c|c|c|}
\hline$\overline{1}$ & 2 & 3 & $\overline{4}$ & $\overline{5}$ & $\overline{6}$ & & 7 & $\overline{8}$ & $\overline{99}$ & $\overline{10}$ \\
\hline $216.3-04.4$ & We $1-5$ & s 5 & & $1.9 \times 1.7$ & & & $1.6 \times 1.5$ & $20.6 \times 19.5$ & & \\
\hline $221.3-12.3$ & IC 2165 & s 4 & $5.8 \times 5.0$ & $2.2 \times 2.1$ & $5.4 \mathrm{x}$ & 5.0 & $1.9 \times 1.8$ & $10.1 \times 9.7$ & & \\
\hline $221.7+05.3$ & M 3- 3 & & & $2.0 \times 2.2$ & $9.3 \mathrm{x}$ & 8.3 & $2.0 \times 2.2$ & $17.1 \times 16.2$ & & \\
\hline $224.9+01.0$ & We 1-6 & s 8 & & $2.1 \times 2.0$ & & & $1.9 \times 1.8$ & $61.0 \times 58.5$ & & \\
\hline $226.4-03.7$ & PB 1 & g 32 & $5.8 \times 5.5$ & $1.9 \times 2.3$ & $4.9 \mathrm{x}$ & 4.8 & $1.9 \times 2.3$ & $11.2 \times 10.4$ & & \\
\hline $226.7+05.6$ & M 1-16 & s 9 & $3.9 \times 3.1$ & $2.4 \times 2.3$ & $5.3 \mathrm{x}$ & 4.0 & $2.3 \times 2.2$ & $8.8 \times \quad 7.0$ & $96.6 \times 19.6$ & 0.1 \\
\hline $228.8+05.3$ & M 1-17 & g 27 & $2.1 \times 1.8$ & $2.3 \times 2.1$ & $2.7 \mathrm{x}$ & 2.4 & $2.1 \times 1.9$ & $5.8 \times 5.4$ & & \\
\hline $229.6-02.7$ & K 1-10 & s 8 & & $1.0 \times 0.8$ & & & $1.3 \times 1.1$ & $51.3 \times 46.0$ & $90.5 \times 60.4$ & 3.6 \\
\hline $231.4+04.3$ & M 1-18 & s 10 & & $1.5 \times 1.7$ & $19.1 \times 1$ & 18.3 & $1.6 \times 1.9$ & $35.0 \times 33.0$ & & \\
\hline $231.8+04.1$ & NGC 2438 & s 9 & & $1.1 \times 1.0$ & & & $1.5 \times 1.4$ & $80.7 \times 78.3$ & & \\
\hline 232.4-01.8 & M 1-13 & g 26 & & $1.5 \times 1.7$ & $9.5 \mathrm{x}$ & 6.9 & $2.1 \times 2.2$ & $18.8 \times 12.0$ & & \\
\hline $232.8-04.7$ & M 1-11 & s 7 & $3.2 \times 2.9$ & $1.1 \times 1.0$ & & & $1.3 \times 1.2$ & $5.5 \times \quad 5.5$ & & \\
\hline $233.5-16.3$ & A 15 & s 5 & & $2.1 \times 1.8$ & & & $1.5 \times 1.3$ & $36.7 \times 34.9$ & & \\
\hline $234.8+02.4$ & NGC 2440 & s 10 & & $1.6 \times 1.4$ & & & $1.6 \times 1.3$ & $58.9 \times 25.3$ & $74.6 \times 55.6$ & 0.2 \\
\hline $234.9-01.4$ & M 1-14 & g 26 & $3.3 \times 2.6$ & $1.7 \times 2.2$ & $3.4 \mathrm{x}$ & 3.0 & $1.7 \times 1.9$ & $6.5 \times 6.5$ & & \\
\hline $236.7+03.5$ & K 1-12 & s 11 & & $1.5 \times 1.4$ & & & $1.2 \times 1.1$ & $44.2 \times 36.5$ & & \\
\hline $238.9+07.3$ & $\mathrm{Sa} 2-21$ & s 12 & & $1.2 \times 0.9$ & & & $1.1 \times 0.9$ & $40.4 \times 34.4$ & & \\
\hline \multirow[t]{2}{*}{$239.6+13.9$} & NGC 2610 & & & $1.4 \times 1.2$ & $25.4 \times 2$ & 23.8 & $1.4 \times 1.4$ & $47.5 \times 46.6$ & & \\
\hline & & s 14 & & $0.9 \times 0.8$ & & & $0.9 \times 0.8$ & $52.0 \times 48.6$ & & \\
\hline 240.3-07.6 & M 3- 2 & g 1 & & $1.8 \times 1.8$ & $7.3 \mathrm{x}$ & 5.8 & $1.9 \times 2.0$ & $12.7 \times 9.7$ & $34.9 \times 15.9$ & 3.0 \\
\hline \multirow[t]{2}{*}{$242.6-11.6$} & M 3- 1 & g 28 & $7.2 \times 4.2$ & $1.7 \times 1.7$ & $7.0 \mathrm{x}$ & 7.7 & $2.0 \times 1.8$ & $13.1 \times 11.6$ & $28.0 \times 17.4$ & 1.1 \\
\hline & & s 6 & $7.3 \times 5.9$ & $1.9 \times 1.8$ & $7.0 \mathrm{x}$ & 6.3 & $1.5 \times 1.6$ & $13.0 \times 10.9$ & $27.8 \times 16.4$ & 2.2 \\
\hline $243.3-01.0$ & NGC 2452 & s 10 & & $1.5 \times 1.4$ & $12.4 \times 1$ & 10.7 & $1.7 \times 1.4$ & $18.5 \times 12.7$ & $33.6 \times 27.6$ & 0.7 \\
\hline $245.4+01.6$ & M 3- 5 & s 11 & $5.0 \times 4.8$ & $1.0 \times 1.2$ & $4.6 \mathrm{x}$ & 4.7 & $0.9 \times 1.1$ & $8.6 \times 7.5$ & & \\
\hline $248.8-08.5$ & M 4- 2 & s 9 & $5.1 \times 3.7$ & $1.8 \times 1.7$ & $4.3 \mathrm{x}$ & 3.6 & $1.7 \times 1.7$ & $8.8 \times \quad 7.8$ & & \\
\hline $249.0+06.9$ & SaSt $1-1$ & s 13 & $0.7 \times 0.7$ & $0.8 \times 0.6$ & $1.1 \mathrm{x}$ & 1.1 & $0.9 \times 0.8$ & $1.8 \times 1.6$ & $13.5 \times 13.0$ & 0.1 \\
\hline $250.3+00.1$ & A 26 & g 12 & & $1.3 \times 1.3$ & & & $1.4 \times 1.4$ & $37.6 \times 36.8$ & & \\
\hline $252.6+04.4$ & K $1-1$ & s 13 & & $0.8 \times 0.8$ & & & $0.8 \times 0.8$ & $51.3 \times 47.5$ & & \\
\hline $258.1-00.3$ & He 2- 9 & s 13 & $3.2 \times 3.2$ & $0.7 \times 0.9$ & $2.9 \mathrm{x}$ & 2.8 & $0.9 \times 0.9$ & $6.0 \times 5.0$ & & \\
\hline $259.1+00.9$ & He 2- 11 & g 13 & & $1.6 \times 1.5$ & & & $1.5 \times 1.2$ & $121.7 \times 64.1$ & & \\
\hline $261.0+32.0$ & NGC 3242 & s 19 & & $1.0 \times 0.7$ & & & $0.9 \times 0.7$ & $31.5 \times 30.7$ & $62.9 \times 54.6$ & 0.1 \\
\hline $261.9+08.5$ & NGC 2818 & g 10 & & $1.4 \times 1.2$ & & & $1.7 \times 1.7$ & $56.2 \times 46.1$ & $118.1 \times 56.5$ & 2.1 \\
\hline $262.6-04.6$ & Wray $17-18$ & s 12 & & $0.9 \times 0.8$ & & & $0.9 \times 0.9$ & $17.3 \times 16.9$ & & \\
\hline 264.4-12.7 & He 2- 5 & s 11 & $2.0 \times 1.8$ & $1.4 \times 1.3$ & $2.2 \mathrm{x}$ & 2.0 & $1.4 \times 1.2$ & $4.4 \times \quad 4.4$ & & \\
\hline $265.7+04.1$ & NGC 2792 & s 15 & & $0.7 \times 0.7$ & $9.7 \mathrm{x}$ & 9.0 & $0.7 \times 0.7$ & $17.9 \times 16.4$ & $25.0 \times 22.1$ & 1.1 \\
\hline $268.4+02.4$ & PB 5 & s 15 & $1.0 \times 1.0$ & $0.7 \times 0.7$ & $1.2 \mathrm{x}$ & 1.0 & $0.7 \times 0.8$ & $2.1 \times 2.1$ & & \\
\hline $272.1+12.3$ & NGC 3132 & s 18 & & $0.8 \times 0.8$ & & & $0.8 \times 0.7$ & $59.9 \times 45.6$ & $86.7 \times 58.5$ & 0.4 \\
\hline $273.2-03.7$ & He 2- 18 & g 14 & $9.3 \times 7.3$ & $2.1 \times 1.8$ & $6.9 \mathrm{x}$ & 7.3 & $2.3 \times 2.1$ & $16.7 \times 14.2$ & & \\
\hline $274.3+09.1$ & Lo 4 & s 17 & & $0.7 \times 0.7$ & & & $0.7 \times 0.7$ & $41.6 \times 38.9$ & & \\
\hline $274.6+02.1$ & He 2- 35 & g 15 & $2.2 \times 2.0$ & $2.0 \times 1.7$ & $2.4 \mathrm{x}$ & 2.3 & $2.0 \times 1.7$ & $5.4 \times \quad 4.7$ & & \\
\hline $274.6+03.5$ & He 2- 37 & g 15 & & $1.8 \times 1.9$ & $13.3 \times 1$ & 13.0 & $2.1 \times 2.2$ & $26.3 \times 22.4$ & & \\
\hline $275.0-04.1$ & PB 4 & s 15 & & $0.8 \times 0.8$ & $6.9 \mathrm{x}$ & 5.3 & $0.9 \times 0.9$ & $12.3 \times 10.3$ & $18.2 \times 13.0$ & 1.3 \\
\hline $275.2-02.9$ & He 2- 28 & g 14 & $5.9 \times 5.9$ & $2.1 \times 2.3$ & $5.1 \mathrm{x}$ & 4.8 & $2.5 \times 2.6$ & $11.5 \times 10.8$ & & \\
\hline $275.2-03.7$ & He 2- 25 & s 16 & & $0.7 \times 0.7$ & & & $0.8 \times 0.8$ & $54.2 \times 10.7$ & & \\
\hline 275.8-02.9 & He 2- 29 & g 14 & $7.8 \times 4.5$ & $2.2 \times 1.8$ & $8.7 \mathrm{x}$ & 7.7 & $2.4 \times 2.1$ & $16.3 \times 12.5$ & $19.4 \times 15.9$ & 1.9 \\
\hline $277.1-03.8$ & NGC 2899 & g 11 & & $1.7 \times 1.5$ & & & $1.9 \times 2.0$ & $68.6 \times 59.9$ & $117.5 \times 66.1$ & 2.1 \\
\hline 278.1-05.9 & NGC 2867 & s 16 & & $0.7 \times 0.8$ & $8.6 \mathrm{x}$ & 8.3 & $0.8 \times 0.8$ & $14.5 \times 14.0$ & $28.3 \times 27.3$ & 0.1 \\
\hline 279.6-03.1 & He 2- 36 & s 17 & & $0.8 \times 0.7$ & $11.1 \mathrm{x}$ & 8.8 & $0.8 \times 0.9$ & $24.8 \times 15.3$ & $31.1 \times 20.0$ & 0.7 \\
\hline $283.8+02.2$ & My 60 & s 19 & & $0.8 \times 0.6$ & $5.3 \mathrm{x}$ & 5.1 & $0.8 \times 0.7$ & $10.2 \times 10.2$ & $13.0 \times 12.8$ & 1.0 \\
\hline $283.8-04.2$ & He 2- 39 & g 15 & $8.3 \times 7.0$ & $2.1 \times 1.8$ & $6.2 \mathrm{x}$ & 6.0 & $2.2 \times 1.9$ & $13.0 \times 12.6$ & & \\
\hline 283.9-01.8 & Hf 4 & s 18 & & $0.8 \times 0.8$ & & & $0.8 \times 0.8$ & $29.1 \times 21.0$ & $40.2 \times 29.4$ & 3.8 \\
\hline $285.4+02.2$ & Pe 2- 7 & g 33 & $3.0 \times 2.0$ & $1.8 \times 2.1$ & $2.7 \mathrm{x}$ & 2.1 & $1.9 \times 2.0$ & $6.5 \times \quad 5.8$ & & \\
\hline $285.4-05.3$ & IC 2553 & s 18 & $5.4 \times 4.4$ & $0.7 \times 0.9$ & $5.7 \mathrm{x}$ & 4.1 & $0.8 \times 0.9$ & $11.6 \times 7.6$ & $19.5 \times 16.6$ & 0.1 \\
\hline $285.6-02.7$ & He 2- 47 & g 16 & $2.7 \times 2.6$ & $2.1 \times 2.4$ & $3.4 \mathrm{x}$ & 3.6 & $2.0 \times 2.3$ & $6.5 \times \quad 5.8$ & & \\
\hline $285.7+01.2$ & Pe 1- 2 & s 20 & $2.0 \times 1.9$ & $0.7 \times 0.8$ & $2.0 \mathrm{x}$ & 3.0 & $0.8 \times 0.8$ & $4.2 \times 3.4$ & $15.6 \times \quad 7.6$ & 0.2 \\
\hline $285.7-14.9$ & IC 2448 & s 14 & $5.5 \times 4.7$ & $0.5 \times 0.5$ & $5.5 \mathrm{x}$ & 5.4 & $0.5 \times 0.5$ & $10.7 \times 10.0$ & & \\
\hline $286.3-04.8$ & NGC 3211 & g 31 & $9.7 \times 7.7$ & $2.4 \times 2.6$ & $8.5 \mathrm{x}$ & 8.4 & $2.5 \times 2.6$ & $16.7 \times 16.6$ & & \\
\hline 288.4-02.4 & Pe 1- 3 & g 32 & $5.9 \times 5.5$ & $2.0 \times 2.3$ & $4.5 \mathrm{x}$ & 5.2 & $2.2 \times 2.5$ & $11.5 \times 9.8$ & & \\
\hline $289.8+07.7$ & He 2- 63 & g 16 & $1.7 \times 1.4$ & $2.0 \times 1.8$ & $1.8 \mathrm{x}$ & 1.6 & $1.9 \times 1.8$ & $4.7 \times \quad 4.5$ & & \\
\hline
\end{tabular}


Table 1. continued.

\begin{tabular}{|c|c|c|c|c|c|c|c|c|c|c|c|}
\hline 1 & 2 & 3 & 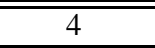 & $\overline{5}$ & 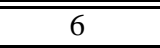 & 7 & $\overline{8}$ & & $\overline{9}$ & & 10 \\
\hline $291.4+19.2$ & ESO 320-28 & s 22 & & $1.2 \times 0.9$ & & $1.0 \times 0.9$ & $30.4 \mathrm{x}$ & 27.3 & & & \\
\hline $291.6-04.8$ & IC 2621 & s 20 & $2.6 \times 2.2$ & $1.0 \times 0.9$ & $2.7 \times 2.3$ & $1.0 \times 0.9$ & $4.4 \mathrm{x}$ & 3.9 & & & \\
\hline $291.7+03.7$ & He 2- 64 & s 21 & $5.6 \times 3.5$ & $0.9 \times 0.9$ & $6.0 \times 7.1$ & $1.0 \times 1.0$ & $9.2 \mathrm{x}$ & 8.5 & $21.4 \mathrm{x}$ & 11.8 & 2.4 \\
\hline $292.4+04.1$ & PB 8 & s 21 & $4.1 \times 3.7$ & $0.9 \times 1.0$ & $3.6 \times 3.7$ & $0.9 \times 1.0$ & $6.8 \mathrm{x}$ & 6.8 & & & \\
\hline $292.6+01.2$ & NGC 3699 & g 11 & & $1.4 \times 1.2$ & & $1.5 \times 1.4$ & $47.0 \mathrm{x}$ & 37.1 & $62.9 x$ & 54.0 & 3.8 \\
\hline $292.8+01.1$ & He 2- 67 & g 2 & $2.5 \times 1.9$ & $1.9 \times 1.9$ & $3.8 \times 2.6$ & $1.9 \times 1.9$ & $6.2 \mathrm{x}$ & 4.4 & $19.4 \mathrm{x}$ & 12.8 & 0.3 \\
\hline $293.6+01.2$ & He 2- 70 & g 3 & & $1.3 \times 1.2$ & & $1.4 \times 1.4$ & $34.7 \mathrm{x}$ & 13.8 & $74.6 \mathrm{x}$ & 51.9 & 1.7 \\
\hline $294.1+14.4$ & Lo 6 & s 22 & & $1.1 \times 1.0$ & & $1.1 \times 1.0$ & $77.0 \mathrm{x}$ & 74.4 & & & \\
\hline $294.6+04.7$ & NGC 3918 & s 22 & $10.7 \times 9.9$ & $1.0 \times 1.1$ & $9.1 \times 9.3$ & $1.0 \times 1.1$ & $18.8 \mathrm{x}$ & 17.2 & $48.5 \mathrm{x}$ & 43.7 & 0.1 \\
\hline 294.9-04.3 & He 2- 68 & g 16 & $1.3 \times 1.3$ & $1.8 \times 1.7$ & $2.2 \times 2.1$ & $1.8 \times 1.7$ & $4.3 \times$ & 4.0 & & & \\
\hline 296.3-03.0 & He 2- 73 & s 21 & $1.7 \times 1.5$ & $1.1 \times 1.0$ & $2.1 \times \quad 1.7$ & $1.1 \times 1.1$ & $3.9 \mathrm{x}$ & 3.1 & & & \\
\hline $296.6-20.0$ & NGC 3195 & g 2 & & $1.5 \times 1.6$ & $20.4 \times 20.2$ & $1.6 \times 1.7$ & $39.6 \mathrm{x}$ & 33.9 & & & \\
\hline $297.4+03.7$ & He 2- 78 & g 17 & $1.9 \times 1.9$ & $2.4 \times 1.9$ & $2.4 \times \quad 2.2$ & $2.4 \times 2.1$ & $5.4 \mathrm{x}$ & 5.0 & & & \\
\hline 298.1-00.7 & He 2- 77 & g 17 & $10.0 \times 6.6$ & $1.8 \times 1.9$ & $14.4 \times 8.0$ & $2.2 \times 2.2$ & $25.6 \mathrm{x}$ & 14.5 & $47.4 \mathrm{x}$ & 32.1 & 2.4 \\
\hline $298.2-01.7$ & He 2- 76 & g 3 & $6.5 \times 4.9$ & $1.8 \times 1.9$ & $7.9 \times 11.0$ & $2.0 \times 2.1$ & $17.4 \mathrm{x}$ & 17.3 & $40.7 \mathrm{x}$ & 21.3 & 4.6 \\
\hline $298.3-04.8$ & NGC 4071 & g 31 & & $1.5 \times 1.3$ & & $1.6 \times 1.5$ & $52.7 \mathrm{x}$ & 72.4 & & & \\
\hline $299.0+18.4$ & K 1-23 & s 23 & & $1.2 \times 1.2$ & & $1.1 \times 1.0$ & $64.3 \times$ & 56.4 & & & \\
\hline $299.5+02.4$ & He 2- 82 & g 4 & & $1.8 \times 1.7$ & $15.2 \times 14.7$ & $1.7 \times 1.9$ & $32.0 \mathrm{x}$ & 25.6 & & & \\
\hline $299.8-01.3$ & He 2- 81 & g 17 & $4.2 \times 3.7$ & $1.7 \times 1.7$ & $3.7 \times 3.4$ & $1.6 \times 1.7$ & $7.9 x$ & 7.2 & & & \\
\hline $300.2+00.6$ & He $2-83$ & g 18 & $2.7 \times 2.4$ & $1.9 \times 2.0$ & $2.8 \times 3.0$ & $1.9 \times 1.9$ & $5.8 \mathrm{x}$ & 5.8 & & & \\
\hline 300.4-00.9 & He 2- 84 & g 4 & & $1.4 \times 1.1$ & & $1.3 \times 1.2$ & $35.9 x$ & 23.8 & & & \\
\hline $300.5-01.1$ & He 2- 85 & g 18 & $5.2 \times 3.4$ & $2.0 \times 2.1$ & $4.4 \times \quad 4.9$ & $2.0 \times 2.1$ & $10.0 \mathrm{x}$ & 8.7 & & & \\
\hline $300.7-02.0$ & He 2- 86 & g 18 & $2.0 \times 1.2$ & $2.0 \times 1.9$ & $3.0 \times \quad 1.7$ & $2.1 \times 1.9$ & $5.6 \mathrm{x}$ & 4.3 & & & \\
\hline $304.5-04.8$ & IC 4191 & s 23 & $3.3 \times 2.9$ & $0.9 \times 1.1$ & $3.8 \times 3.9$ & $0.9 \times 1.1$ & $5.6 x$ & 4.9 & $23.0 \mathrm{x}$ & 20.5 & 0.1 \\
\hline $304.8+05.1$ & He 2- 88 & g 19 & $0.7 \times 0.7$ & $2.6 \times 1.7$ & $1.7 \times \quad 1.6$ & $2.4 \times 1.7$ & $4.7 \mathrm{x}$ & 4.0 & & & \\
\hline $305.1+01.4$ & He 2- 90 & s 24 & $1.7 \times 1.6$ & $1.2 \times 1.0$ & $2.5 \times 2.3$ & $1.2 \times 1.1$ & $3.9 \mathrm{x}$ & 3.6 & & & \\
\hline $306.4-00.6$ & Th 2- A & g 34 & & $2.5 \times 2.4$ & $15.8 \times 13.2$ & $2.7 \times 2.6$ & $27.7 x$ & 25.2 & & & \\
\hline $307.2-03.4$ & NGC 5189 & g 11 & & $1.7 \times 1.6$ & & $2.2 \times 1.9$ & $163.4 \times 1$ & 108.2 & $177.6 \mathrm{x}$ & 130.9 & 2.1 \\
\hline $307.2-09.0$ & He 2- 97 & s 25 & $1.4 \times 1.2$ & $1.3 \times 1.1$ & $1.6 \times 1.5$ & $1.2 \times 1.1$ & $3.6 \mathrm{x}$ & 2.6 & & & \\
\hline $307.5-04.9$ & MyCn 18 & s 24 & & $1.4 \times 1.2$ & & $1.4 \times 1.3$ & $17.4 \times$ & 10.1 & $23.7 \mathrm{x}$ & 17.7 & 0.5 \\
\hline $308.6-12.2$ & He 2-105 & s 27 & & $1.3 \times 1.1$ & & $1.1 \times 1.1$ & $41.6 \mathrm{x}$ & 40.7 & & & \\
\hline $309.0+00.8$ & He 2- 96 & g 19 & $1.5 \times 1.3$ & $2.1 \times 2.0$ & $2.8 \times 2.2$ & $2.1 \times 2.1$ & $4.7 \mathrm{x}$ & 4.7 & & & \\
\hline $309.0-04.2$ & He 2- 99 & s 25 & & $1.3 \times 1.0$ & $13.3 \times 12.1$ & $1.3 \times 1.1$ & $28.0 \mathrm{x}$ & 23.5 & & & \\
\hline $309.1-04.3$ & NGC 5315 & s 25 & $6.0 \times 5.9$ & $1.0 \times 1.1$ & $5.7 \times 5.9$ & $1.1 \times 1.2$ & $10.9 \mathrm{x}$ & 9.4 & $50.7 \mathrm{x}$ & 44.0 & 0.1 \\
\hline $310.7-02.9$ & He 2-103 & g 20 & & $1.5 \times 1.7$ & $11.4 \times 11.0$ & $1.8 \times 1.9$ & $22.3 x$ & 21.1 & & & \\
\hline $311.0+02.4$ & SuWt 2 & s 26 & & $1.0 \times 0.8$ & & $1.0 \times 0.9$ & $86.5 \mathrm{x}$ & 43.4 & & & \\
\hline $311.4+02.8$ & He 2-102 & g 19 & $6.5 \times 5.6$ & $2.2 \times 2.1$ & $5.4 \times \quad 5.8$ & $2.5 \times 2.5$ & $12.3 \mathrm{x}$ & 12.0 & & & \\
\hline $312.3+10.5$ & NGC 5307 & g 31 & $12.0 \times 6.7$ & $2.4 \times 2.3$ & $9.7 \times \quad 6.7$ & $2.9 \times 2.8$ & $19.3 \mathrm{x}$ & 13.6 & & & \\
\hline $312.6-01.8$ & He 2-107 & s 28 & $7.2 \times 5.1$ & $1.3 \times 1.2$ & $6.0 \times 4.7$ & $1.5 \times 1.5$ & $11.0 \mathrm{x}$ & 8.6 & & & \\
\hline $315.0-00.3$ & He 2-111 & g 20 & & $1.8 \times 1.7$ & $20.2 \times 12.0$ & $2.2 \times 2.1$ & $29.6 \mathrm{x}$ & 14.8 & $106.6 \mathrm{x}$ & 52.7 & 0.5 \\
\hline $315.1-13.0$ & He 2-131 & s 33 & & $1.1 \times 1.0$ & & $1.1 \times 1.1$ & $10.2 \mathrm{x}$ & 9.8 & & & \\
\hline $315.4+05.2$ & He 2-109 & g 20 & $5.6 \times 5.5$ & $1.9 \times 1.8$ & $5.2 \times 4.2$ & $2.0 \times 2.0$ & $11.5 \mathrm{x}$ & 8.2 & & & \\
\hline $315.4+09.4$ & He 2-104 & s 26 & & $1.4 \times 1.0$ & & $1.3 \times 1.1$ & $85.6 \mathrm{x}$ & 34.4 & & & \\
\hline $315.7+05.5$ & LoTr 8 & s 28 & & $1.1 \times 1.0$ & & $1.3 \times 1.2$ & $28.5 \mathrm{x}$ & 25.2 & & & \\
\hline $316.1+08.4$ & He 2-108 & s 27 & & $1.4 \times 1.1$ & $7.3 \times \quad 6.8$ & $1.3 \times 1.2$ & $13.8 \mathrm{x}$ & 12.5 & & & \\
\hline $317.1-05.7$ & He $2-119$ & g 5 & & $1.8 \times 1.7$ & & $1.7 \times 1.6$ & $63.4 \mathrm{x}$ & 60.5 & $108.6 \mathrm{x}$ & 68.9 & 2.5 \\
\hline $318.3-02.0$ & He $2-114$ & g 4 & & $1.7 \times 1.8$ & $13.7 \times 13.2$ & $1.9 \times 1.9$ & $26.3 \times$ & 21.6 & & & \\
\hline $318.3-02.5$ & He 2-116 & g 5 & & $3.3 \times 2.9$ & $27.3 \times 26.2$ & $3.2 \times 2.9$ & $48.2 \mathrm{x}$ & 47.1 & & & \\
\hline $319.2+06.8$ & He 2-112 & g 21 & $3.9 \times 3.9$ & $1.8 \times 1.9$ & $3.8 \times 3.7$ & $1.8 \times 1.9$ & $7.6 \mathrm{x}$ & 7.2 & & & \\
\hline $319.6+15.7$ & IC 4406 & s 28 & & $1.3 \times 1.2$ & & $1.5 \times 1.5$ & $46.5 \mathrm{x}$ & 30.0 & $103.7 \mathrm{x}$ & 39.3 & 0.7 \\
\hline $320.1-09.6$ & He $2-138$ & g 22 & $3.9 \times 3.7$ & $1.7 \times 1.8$ & $4.0 \times 3.8$ & $1.6 \times 1.8$ & $7.3 \mathrm{x}$ & 6.8 & & & \\
\hline $320.3-28.8$ & He 2-434 & s 82 & $3.5 \times 2.4$ & $1.2 \times 0.9$ & $3.6 \times 2.6$ & $1.0 \times 0.8$ & $7.7 \mathrm{x}$ & 5.3 & & & \\
\hline $320.9+02.0$ & He 2-117 & s 29 & $2.9 \times 2.9$ & $0.7 \times 0.6$ & $3.1 \times 2.8$ & $0.7 \times 0.7$ & $5.5 \mathrm{x}$ & 4.5 & & & \\
\hline \multirow[t]{2}{*}{$321.3+02.8$} & He $2-115$ & g 21 & $1.8 \times 1.5$ & $1.3 \times 1.2$ & $2.3 \times 2.0$ & $1.3 \times 1.2$ & $4.5 \mathrm{x}$ & 3.1 & & & \\
\hline & & s 29 & $1.6 \times 1.5$ & $0.7 \times 0.7$ & $1.9 \times 1.5$ & $0.9 \times 0.8$ & $3.4 \mathrm{x}$ & 2.4 & & & \\
\hline $321.3-16.7$ & He $2-185$ & s 41 & $1.7 \times 1.3$ & $0.8 \times 0.7$ & $1.6 \times \quad 1.4$ & $0.9 \times 0.9$ & $3.3 \mathrm{x}$ & 2.8 & & & \\
\hline $321.8+01.9$ & He $2-120$ & g 5 & & $1.7 \times 1.7$ & $19.7 \times 17.0$ & $1.8 \times 1.7$ & $36.2 \mathrm{x}$ & 26.7 & & & \\
\hline $322.1-06.6$ & He 2-136 & s 34 & $3.9 \times 3.8$ & $1.2 \times 1.2$ & $3.3 \times 4.2$ & $1.3 \times 1.3$ & $7.6 \mathrm{x}$ & 5.2 & & & \\
\hline $322.4-00.1$ & Pe 2- 8 & g 33 & $1.2 \times 1.0$ & $2.7 \times 2.2$ & $2.2 \times 2.0$ & $2.6 \times 2.1$ & $5.8 \mathrm{x}$ & 4.7 & & & \\
\hline
\end{tabular}


Table 1. continued.

\begin{tabular}{|c|c|c|c|c|c|c|c|c|c|}
\hline$\overline{11}$ & 2 & 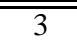 & 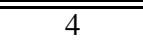 & $\overline{\overline{5}}$ & $\overline{\overline{6}}$ & $\overline{77}$ & $\overline{88}$ & $\overline{\overline{9}}$ & $\overline{10}$ \\
\hline $322.4-02.6$ & $\mathrm{Mz} 1$ & s 32 & & $0.9 \times 0.9$ & & $1.0 \times 1.0$ & $49.3 \times 35.3$ & & \\
\hline $322.5-05.2$ & NGC 5979 & s 33 & & $1.1 \times 1.2$ & $11.5 \times 10.5$ & $1.2 \times 1.3$ & $20.3 \times 19.2$ & $26.6 \times 23.3$ & 1.2 \\
\hline $323.1-02.5$ & He 2-132 & g 22 & & $1.9 \times 1.9$ & $10.8 \times \quad 9.9$ & $2.1 \times 2.1$ & $21.1 \times 19.2$ & & \\
\hline $323.9+02.4$ & He $2-123$ & s 31 & $4.3 \times 3.5$ & $0.9 \times 0.9$ & $4.1 \times 5.9$ & $1.0 \times 1.0$ & $7.1 \times 6.8$ & $34.4 \times 12.2$ & 0.2 \\
\hline $324.2+02.5$ & He $2-125$ & s 31 & $2.0 \times 1.8$ & $0.9 \times 1.0$ & $2.1 \times 1.8$ & $1.0 \times 1.0$ & $4.2 \times 3.4$ & & \\
\hline $324.8-01.1$ & He $2-133$ & s 33 & $1.1 \times 1.0$ & $1.0 \times 1.1$ & $1.6 \times 1.6$ & $1.1 \times 1.2$ & $2.9 \times 2.3$ & & \\
\hline $325.0+03.2$ & He 2-129 & g 21 & $1.7 \times 1.4$ & $1.8 \times 1.7$ & $2.0 \times 1.8$ & $1.9 \times 1.8$ & $4.3 \times 4.3$ & & \\
\hline $325.4-04.0$ & He $2-141$ & s 35 & & $0.9 \times 1.0$ & $9.9 \times \quad 7.3$ & $1.0 \times 1.0$ & $13.1 \times 10.9$ & $33.3 \times 16.0$ & 0.6 \\
\hline $325.8+04.5$ & He $2-128$ & s 32 & $1.2 \times 1.0$ & $0.9 \times 0.9$ & $1.4 \times \quad 1.2$ & $0.9 \times 1.0$ & $2.3 \times 2.1$ & & \\
\hline $325.8-12.8$ & He $2-182$ & s 41 & $1.9 \times 1.9$ & $0.7 \times 0.8$ & $2.2 \times 2.0$ & $0.9 \times 0.9$ & $3.4 \times \quad 3.1$ & & \\
\hline $326.0-06.5$ & He $2-151$ & s 37 & $1.0 \times 0.9$ & $0.8 \times 0.8$ & $1.2 \times \quad 1.1$ & $0.9 \times 0.9$ & $2.3 \times 2.3$ & & \\
\hline $327.1-01.8$ & He $2-140$ & g 22 & $2.4 \times 2.1$ & $2.5 \times 2.0$ & $2.8 \times 2.6$ & $2.6 \times 2.3$ & $6.2 \times 5.4$ & & \\
\hline $327.1-02.2$ & He $2-142$ & s 35 & $2.4 \times 2.2$ & $0.9 \times 0.8$ & $2.4 \times 2.1$ & $0.9 \times 0.9$ & $4.4 \times 3.5$ & & \\
\hline $327.5+13.3$ & He $2-118$ & s 30 & $1.0 \times 0.7$ & $0.7 \times 0.7$ & $1.0 \times \quad 0.8$ & $0.8 \times 0.7$ & $1.8 \times 1.6$ & & \\
\hline $327.8+10.0$ & NGC 5882 & s 30 & & $0.7 \times 0.7$ & $7.8 \times \quad 6.9$ & $0.7 \times 0.7$ & $15.6 \times 13.0$ & & \\
\hline $327.8-01.6$ & He $2-143$ & g 23 & $2.4 \times 1.5$ & $2.4 \times 2.4$ & $3.1 \times 2.0$ & $2.5 \times 2.5$ & $6.5 \times \quad 5.1$ & & \\
\hline $327.8-06.1$ & He $2-158$ & s 38 & $1.3 \times 0.9$ & $1.3 \times 1.4$ & $2.0 \times 1.5$ & $1.4 \times 1.5$ & $3.4 \times 2.9$ & & \\
\hline $327.8-07.2$ & He $2-163$ & g 24 & & $1.8 \times 1.7$ & $12.8 \times 10.8$ & $2.0 \times 1.9$ & $22.3 \times 22.0$ & & \\
\hline $327.9-04.3$ & He 2-147 & g 23 & $2.1 \times 1.3$ & $1.8 \times 1.6$ & $2.4 \times \quad 1.6$ & $1.8 \times 1.7$ & $5.8 \times 4.7$ & & \\
\hline $328.2+01.3$ & Lo 10 & s 34 & & $1.3 \times 1.2$ & & $1.2 \times 1.2$ & $28.2 \times 25.3$ & & \\
\hline $328.9-02.4$ & He 2-146 & g 6 & & $1.5 \times 1.2$ & & $1.7 \times 1.6$ & $38.5 \times 30.6$ & & \\
\hline $330.6-02.1$ & He $2-153$ & g 7 & & $1.8 \times 1.6$ & $10.5 \times 8.8$ & $2.0 \times 2.0$ & $19.1 \times 13.5$ & & \\
\hline $330.6-03.6$ & He 2-159 & g 23 & $6.9 \times 5.2$ & $1.6 \times 1.4$ & $7.8 \times \quad 4.7$ & $1.7 \times 1.7$ & $15.5 \times 10.8$ & & \\
\hline $330.7+04.1$ & Cn 1-1 & g 12 & st. & $2.4 \times 1.7$ & $1.8 \times 1.7$ & $2.4 \times 1.8$ & $4.3 \times 2.9$ & & \\
\hline $331.3+16.8$ & NGC 5873 & s 30 & $4.8 \times 2.8$ & $0.6 \times 0.7$ & $3.4 \times \quad 4.0$ & $0.8 \times 0.8$ & $7.2 \times 5.2$ & $12.8 \times 12.2$ & 0.1 \\
\hline $331.4+00.5$ & He $2-145$ & g 6 & & $1.6 \times 1.5$ & $9.8 \times \quad 9.5$ & $1.9 \times 1.7$ & $17.0 \times 15.7$ & $24.5 \times 18.3$ & 4.7 \\
\hline $331.5-02.7$ & He 2-161 & g 7 & $6.7 \times 4.7$ & $1.7 \times 1.8$ & $5.6 \times 8.3$ & $1.9 \times 1.9$ & $16.6 \times 10.2$ & & \\
\hline $331.5-03.9$ & He $2-165$ & g 8 & & $1.5 \times 1.5$ & $29.7 \times 25.5$ & $1.9 \times 1.7$ & $56.5 \times 46.4$ & & \\
\hline $331.7-01.0$ & Mz 3 & s 37 & & $0.9 \times 0.8$ & & $0.9 \times 0.8$ & $47.8 \times 22.6$ & & \\
\hline $332.0-03.3$ & He $2-164$ & g 8 & $10.1 \times 8.6$ & $1.5 \times 1.4$ & $8.2 \times 9.4$ & $1.6 \times 1.8$ & $16.6 \times 15.5$ & & \\
\hline $332.3-04.2$ & He $2-170$ & s 39 & $0.7 \times 0.6$ & $0.8 \times 0.9$ & $0.9 \times \quad 0.8$ & $0.9 \times 0.9$ & $2.1 \times 1.8$ & & \\
\hline 332.9-09.9 & He 3-1333 & s 44 & $1.8 \times 1.6$ & $1.1 \times 0.9$ & $2.4 \times \quad 2.1$ & $1.2 \times 1.1$ & $3.6 \times 3.4$ & & \\
\hline $333.4+01.1$ & He 2-152 & g 6 & $6.1 \times 6.1$ & $1.8 \times 1.7$ & $7.6 \times \quad 6.9$ & $1.9 \times 2.0$ & $12.2 \times 10.5$ & & \\
\hline $334.3-09.3$ & IC 4642 & s 45 & & $1.2 \times 0.9$ & $12.2 \times 11.1$ & $1.0 \times 1.0$ & $24.2 \times 21.8$ & & \\
\hline $334.8-07.4$ & SaSt 2-12 & s 42 & & $0.7 \times 0.6$ & & $0.8 \times 0.7$ & $16.0 \times 11.9$ & & \\
\hline $335.4+09.2$ & ESO 330-02 & s 34 & & $1.2 \times 1.1$ & & $1.2 \times 1.2$ & $30.9 \times 28.9$ & & \\
\hline $335.4-01.1$ & He 2-169 & g 8 & & $1.7 \times 1.6$ & & $1.9 \times 1.7$ & $22.5 \times 18.7$ & $29.6 \times 23.4$ & 4.8 \\
\hline $336.2+01.9$ & Pe $1-6$ & g 33 & $6.2 \times 4.3$ & $1.7 \times 1.5$ & $4.7 \times \quad 4.6$ & $1.7 \times 1.7$ & $10.6 \times 9.2$ & & \\
\hline $336.2-06.9$ & PC 14 & s 43 & $3.3 \times 2.8$ & $0.9 \times 0.8$ & $3.0 \times 3.9$ & $1.0 \times 0.9$ & $7.3 \times \quad 5.4$ & & \\
\hline $336.3-05.6$ & He $2-186$ & s 41 & $1.5 \times 1.0$ & $0.6 \times 0.8$ & $1.8 \times 1.7$ & $0.7 \times 0.8$ & $3.1 \times 2.1$ & $10.7 \times 7.3$ & 0.1 \\
\hline $336.8-07.2$ & K 2-17 & s 45 & & $1.1 \times 0.9$ & & $1.3 \times 1.1$ & $39.3 \times 32.5$ & & \\
\hline $337.4+01.6$ & Pe 1- 7 & s 39 & $1.4 \times 1.2$ & $1.2 \times 1.3$ & $2.0 \times 2.0$ & $1.3 \times 1.3$ & $3.4 \times \quad 3.1$ & & \\
\hline $338.1-08.3$ & NGC 6326 & s 46 & & $1.0 \times 1.0$ & $11.8 \times \quad 9.1$ & $1.2 \times 1.3$ & $20.7 \times 13.8$ & $38.5 \times 23.7$ & 0.7 \\
\hline $338.8+05.6$ & He 2-155 & g 7 & $11.9 \times 8.7$ & $1.3 \times 1.6$ & $9.2 \times 8.5$ & $1.5 \times 1.6$ & $17.1 \times 14.7$ & $24.2 \times 19.5$ & 3.2 \\
\hline $340.8+10.8$ & Lo 12 & g 25 & & $1.3 \times 1.3$ & & $1.6 \times 1.5$ & $84.5 \times 70.4$ & & \\
\hline $340.8+12.3$ & Lo 11 & s 36 & & $0.9 \times 0.8$ & & $0.9 \times 0.9$ & $65.7 \times 57.0$ & & \\
\hline $341.6+13.7$ & NGC 6026 & s 35 & & $0.8 \times 0.7$ & & $0.8 \times 0.8$ & $53.0 \times 45.5$ & & \\
\hline $341.8+05.4$ & NGC 6153 & g 32 & & $2.6 \times 1.9$ & $15.2 \times 14.0$ & $2.6 \times 2.2$ & $28.4 \times 24.5$ & & \\
\hline $342.1+10.8$ & NGC 6072 & s 36 & & $0.8 \times 0.7$ & & $0.8 \times 0.8$ & $74.3 \times 65.1$ & & \\
\hline $342.1+27.5$ & Me 2-1 & s 31 & $4.9 \times 4.9$ & $1.1 \times 0.9$ & $4.5 \times \quad 4.4$ & $1.1 \times 1.0$ & $9.1 \times 8.8$ & & \\
\hline $342.9-02.0$ & Pe 1- 8 & g 12 & & $1.8 \times 1.0$ & & $1.7 \times 1.3$ & $24.5 \times 22.1$ & & \\
\hline $342.9-04.9$ & He 2-207 & g 24 & & $1.6 \times 1.4$ & & $1.8 \times 1.7$ & $37.8 \times 26.1$ & & \\
\hline $343.4+11.9$ & H $1-1$ & s 36 & $1.6 \times 1.4$ & $0.8 \times 0.8$ & $1.6 \times \quad 1.5$ & $0.9 \times 0.9$ & $3.4 \times \quad 3.1$ & & \\
\hline $343.6+03.7$ & SuWt 3 & s 39 & & $0.8 \times 0.8$ & & $0.9 \times 0.8$ & $31.9 \times 16.4$ & & \\
\hline $345.2-01.2$ & H 1- 7 & s 45 & $5.7 \times 5.2$ & $1.0 \times 0.7$ & $5.2 \times 6.0$ & $1.0 \times 0.9$ & $10.7 \times 8.9$ & & \\
\hline $345.2-08.8$ & Tc 1 & s 53 & $8.1 \times 7.1$ & $1.9 \times 1.6$ & $6.8 \times 6.4$ & $1.7 \times 1.5$ & $13.3 \times 12.5$ & $52.0 \times 51.0$ & 0.4 \\
\hline $345.4+00.1$ & IC 4637 & s 43 & & $0.8 \times 0.8$ & $11.4 \times 10.0$ & $0.9 \times 0.9$ & $19.0 \times 13.6$ & $30.2 \times 25.5$ & 0.5 \\
\hline $346.2-08.2$ & IC 4663 & s 52 & & $1.6 \times 1.7$ & $10.4 \times \quad 8.7$ & $1.6 \times 1.7$ & $20.3 \times 14.9$ & & \\
\hline $348.0-13.8$ & IC 4699 & s 67 & $8.4 \times 3.5$ & $1.7 \times 1.8$ & $7.0 \times 3.9$ & $1.7 \times 1.8$ & $13.0 \times 8.7$ & & \\
\hline
\end{tabular}


R. Tylenda et al.: Angular dimensions of planetary nebulae, Online Material p 7

Table 1. continued.

\begin{tabular}{|c|c|c|c|c|c|c|c|c|c|c|}
\hline 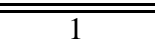 & $\overline{2}$ & 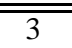 & 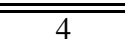 & $\overline{5}$ & 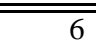 & & $\overline{7}$ & $\overline{88}$ & $\overline{99}$ & $\overline{10}$ \\
\hline $349.3-01.1$ & NGC 6337 & s 47 & & $1.0 \times 0.9$ & & & $1.1 \times 1.0$ & $47.6 \times 46.5$ & & \\
\hline $349.5+01.0$ & NGC 6302 & s 46 & & $0.8 \times 0.7$ & & & $0.9 \times 0.8$ & $89.9 \times 34.8$ & & \\
\hline $350.9+04.4$ & H 2- 1 & s 42 & $2.3 \times 2.2$ & $0.8 \times 0.7$ & $2.5 \mathrm{x}$ & 2.5 & $0.9 \times 0.8$ & $4.5 \times \quad 3.9$ & & \\
\hline $352.9+11.4$ & K 2-16 & s 40 & & $0.8 \times 0.7$ & & & $0.9 \times 0.8$ & $26.6 \times 24.3$ & & \\
\hline $355.1-06.9$ & M 3-21 & s 59 & $1.7 \times 1.4$ & $1.5 \times 1.8$ & $2.6 \mathrm{x}$ & 1.9 & $1.6 \times 1.8$ & $4.4 \times 3.9$ & $15.5 \times 13.7$ & 0.1 \\
\hline $355.4-04.0$ & Hf $2-1$ & g 9 & $9.5 \times 7.0$ & $2.0 \times 1.6$ & $10.4 \mathrm{x}$ & 8.5 & $2.1 \times 1.9$ & $18.1 \times 14.9$ & & \\
\hline $356.5-02.3$ & M 1-27 & s 54 & $4.0 \times 4.0$ & $1.6 \times 1.5$ & $3.6 \mathrm{x}$ & 3.6 & $1.7 \times 1.4$ & $7.3 \times \quad 7.0$ & & \\
\hline $356.7-06.4$ & H $1-51$ & g 3 & & $1.9 \times 1.7$ & $9.2 \mathrm{x}$ & 9.0 & $2.0 \times 1.6$ & $18.0 \times 15.5$ & & \\
\hline $357.0+02.4$ & M 4- 4 & s 48 & $3.4 \times 1.7$ & $0.8 \times 0.8$ & $3.2 \mathrm{x}$ & 3.5 & $0.8 \times 0.8$ & $6.4 \times 5.3$ & & \\
\hline $357.1-04.7$ & H 1-43 & g 13 & $1.0 \times 0.8$ & $2.0 \times 1.9$ & $2.0 \mathrm{x}$ & 1.8 & $1.7 \times 1.7$ & $4.3 \times 4.0$ & & \\
\hline $357.3+04.0$ & Н 2- 7 & s 47 & $2.8 \times 2.6$ & $1.0 \times 0.9$ & $2.7 \mathrm{x}$ & 2.4 & $1.1 \times 1.0$ & $5.9 \times \quad 4.8$ & & \\
\hline $357.6-03.3$ & Н 2-29 & s 56 & $9.9 \times 5.9$ & $1.0 \times 1.1$ & $6.0 \mathrm{x}$ & 5.9 & $1.0 \times 1.1$ & $10.9 \times 10.0$ & & \\
\hline $357.9-03.8$ & H 2-30 & s 57 & $7.3 \times 6.2$ & $1.4 \times 1.3$ & $6.2 \mathrm{x}$ & 4.2 & $1.3 \times 1.2$ & $13.5 \times 13.5$ & & \\
\hline $358.3-21.6$ & IC 1297 & s 79 & $7.3 \times 3.5$ & $0.8 \times 1.0$ & $6.2 \mathrm{x}$ & 6.6 & $0.8 \times 0.9$ & $10.9 \times 9.9$ & $19.0 \times 18.5$ & 0.2 \\
\hline $358.5+05.4$ & M 3-39 & g 29 & & $1.7 \times 1.7$ & 10.6 & 10.3 & $1.6 \times 1.8$ & $26.1 \times 18.4$ & & \\
\hline $358.5-07.3$ & NGC 6563 & s 63 & & $1.5 \times 1.5$ & & & $1.7 \times 1.5$ & $59.2 \times 43.2$ & $80.5 \times 48.0$ & 2.1 \\
\hline $358.6+01.8$ & M 4- 6 & s 50 & $1.5 \times 1.4$ & $1.0 \times 1.0$ & $1.4 \mathrm{x}$ & 1.4 & $1.1 \times 1.1$ & $3.1 \times 2.9$ & & \\
\hline $358.6-05.5$ & M 3-51 & g 10 & & $2.1 \times 1.7$ & $7.8 \mathrm{x}$ & 7.7 & $2.1 \times 2.1$ & $21.2 \times 14.8$ & & \\
\hline 358.9-00.7 & M 1-26 & s 54 & $3.7 \times 3.5$ & $2.1 \times 2.0$ & $3.7 \mathrm{x}$ & 3.6 & $2.2 \times 2.0$ & $7.5 \times \quad 7.0$ & $19.8 \times 19.0$ & 0.1 \\
\hline $359.0-04.1$ & M 3-48 & s 58 & $2.9 \times 2.6$ & $1.4 \times 1.6$ & $3.1 \mathrm{x}$ & 2.5 & $1.5 \times 1.7$ & $6.0 \times 5.2$ & & \\
\hline $359.1+15.1$ & A 40 & s 40 & & $0.9 \times 0.8$ & & & $0.9 \times 0.9$ & $34.3 \times 30.4$ & & \\
\hline $359.2+01.2$ & 19W32 & s 51 & & $1.8 \times 1.6$ & & & $1.7 \times 1.5$ & $23.8 \times 6.4$ & & \\
\hline $359.3-00.9$ & $\mathrm{Hb} 5$ & s 55 & & $1.4 \times 1.2$ & & & $1.6 \times 1.3$ & $51.7 \times 18.3$ & $60.1 \times 27.8$ & 0.9 \\
\hline 359.4-03.4 & H 2-33 & s 58 & $5.3 \times 5.0$ & $1.6 \times 1.4$ & $3.9 \mathrm{x}$ & 3.4 & $1.6 \times 1.3$ & $8.3 \times 7.8$ & & \\
\hline $359.9+05.1$ & M 3- 9 & s 48 & & $0.8 \times 0.8$ & $9.0 \mathrm{x}$ & 7.5 & $0.8 \times 0.9$ & $17.3 \times 15.2$ & & \\
\hline
\end{tabular}

